hep-th/0411280

CALT-68-2529

HUTP-04/A0049

UCB-PTH/04/33

\title{
Black Holes, q-Deformed 2d Yang-Mills, and Non-perturbative Topological Strings
}

\author{
Mina Aganagic, ${ }^{1}$ Hirosi Ooguri, ${ }^{2}$ Natalia Saulina, ${ }^{3}$ and Cumrun Vafa ${ }^{3}$ \\ 1 University of California, Berkeley, CA 94720, USA \\ 2 California Institute of Technology, Pasadena, CA 91125, USA \\ 3 Jefferson Physical Laboratory, Harvard University, Cambridge, MA 02138, USA
}

\begin{abstract}
We count the number of bound states of BPS black holes on local Calabi-Yau threefolds involving a Riemann surface of genus $g$. We show that the corresponding gauge theory on the brane reduces to a $q$-deformed Yang-Mills theory on the Riemann surface. Following the recent connection between the black hole entropy and the topological string partition function, we find that for a large black hole charge $N$, up to corrections of $O\left(e^{-N}\right), Z_{B H}$ is given as a sum of a square of chiral blocks, each of which corresponds to a specific D-brane amplitude. The leading chiral block, the vacuum block, corresponds to the closed topological string amplitudes. The sub-leading chiral blocks involve topological string amplitudes with D-brane insertions at $(2 g-2)$ points on the Riemann surface analogous to the $\Omega$ points in the large $N 2$ d Yang-Mills theory. The finite $N$ amplitude provides a non-perturbative definition of topological strings in these backgrounds. This also leads to a novel non-perturbative formulation of $c=1$ non-critical string at the self-dual radius.
\end{abstract}

November, 2004 


\section{Introduction}

Counting of 4-dimensional BPS black hole microstates arising upon compactifications of type II superstrings on Calabi-Yau 3-folds has been recently connected to topological string amplitudes in a highly non-trivial way [1]. In particular it has been argued that for a large black hole charge $N$ and to all order in $1 / N$ expansion, the mixed ensemble partition function $Z_{B H}$ of BPS black holes is related to topological string amplitudes $Z_{\mathrm{top}}$ :

$$
Z_{B H}=\left|Z_{\mathrm{top}}\right|^{2} .
$$

In fact the proposal in [1] goes further and uses the above relation as defining what one means by the non-perturbative topological string amplitude, including $O\left(e^{-N}\right)$ corrections.

One main obstacle in checking this relation is that both sides are difficult (at present impossible) to compute for compact Calabi-Yau manifolds. However, the logic of [1] can be adapted to non-compact Calabi-Yau manifolds and the counting of BPS states in these geometries. In that context one could hope to check this statement.

A first case where this was actually done [2] was in the case of a local Calabi-Yau involving the sum of two line bundles over a torus $T^{2}$. In that case, not only it was shown that (1.1) is correct to all orders in the $1 / N$ expansion, but that, at finite $N$, the notion of

a holomorphic $Z_{\text {top }}$ ceases to make sense due to $O\left(e^{-N}\right)$ effects. In fact counting of BPS bound states in that case reduces to computing the partition function of the 2d Yang-Mills theory on $T^{2}$, whose large $N$ expansion has the above chiral decomposition valid only to all orders in the $1 / N$ expansion, as was demonstrated in [3].

The aim of this paper is to extend the computation in [2] to the case of a Calabi-Yau manifold where $T^{2}$ is replaced by an arbitrary genus $g$ Riemann surface. Once again we find that the topological gauge theory on the brane reduces to 2d Yang-Mills theory on the Riemann surface, with one additional subtlety: the Yang-Mills theory is q-deformed! We then ask if the relation (11.1) holds in this case. Luckily the topological string amplitude for this geometry has been computed very recently [4], and so the right hand side of (1.1) is also known. In checking this relation we find an interesting new subtlety: We find that the relation (1.1) to all orders in the $1 / N$ expansion is modified in this case to

$$
Z_{B H}=\int \prod_{i=1}^{|2 g-2|} d U_{i}\left|Z_{\mathrm{top}}\left(U_{1}, \ldots, U_{|2 g-2|}\right)\right|^{2},
$$


where $Z_{\text {top }}\left(U_{1}, \ldots, U_{|2 g-2|}\right)$ is the topological string amplitude with $|2 g-2|$ stacks of Dbranes inserted, and $U_{i}$ corresponds to the holonomy of the gauge field turned on in the $i$-th stack of D-branes. The leading piece of this expansion is the closed string amplitude. To extract this piece, the integral over the unitary group in the above can be performed and the relation can be recast as

$$
Z_{B H}=\sum_{R_{1}, \ldots, R_{|2 g-2|}}\left|Z_{R_{1}, \ldots, R_{|2 g-2|}}^{\mathrm{top}}\right|^{2}
$$

where

$$
Z_{\text {top }}\left(U_{1}, \ldots, U_{|2 g-2|}\right)=\sum_{R_{1}, \ldots, R_{|2 g-2|}} Z_{R_{1}, \ldots, R_{|2 g-2|}}^{\text {top }} \operatorname{Tr}_{R_{1}} U_{1} \cdots \operatorname{Tr}_{R_{|2 g-2|}} U_{|2 g-2|}
$$

and $R_{1}, \ldots, R_{|2 g-2|}$ are representations of $S U(\infty)$ and $\operatorname{Tr}_{R_{i}} U_{i}(i=1, \ldots,|2 g-2|)$ are their characters. The closed string amplitude is the vacuum chiral block given by $R_{i}=0$.

The organization of this paper is as follows: In section 2 we review perturbative results for topological strings on a Calabi-Yau with a local geometry involving a Riemann surface. We also present a short alternative derivation of the main result. In section 3 we study the gauge theory living on a BPS brane in this geometry and compute its partition function. In particular we show that the relevant field theory on the brane gets mapped to the $q$-deformed 2d Yang-Mills theory on the Riemann surface. In section 4 we use this result to count the BPS black hole degeneracy in this geometry. In section 5 we take the limit of large black hole charges and relate our result to the expected topological string amplitudes reviewed in section 2, in accordance with [1]. In section 6 we discuss the limit of small area for a local $\mathbf{P}^{1}$ geometry (the resolved conifold). This is related by the mirror symmetry to the deformed conifold and thus to the $c=1$ non-critical bosonic string at the self-dual radius. There we find that the non-perturbative formulation involves chiral blocks which represent a condensation of a coherent state of tachyons which emits tachyons at all frequencies. In appendices A - D we collect some identities needed in the paper.

\section{Perturbative Topological String Theory}

In a recent work [4] topological A-model string theory amplitudes

$$
X=L_{1} \oplus L_{2} \rightarrow \Sigma,
$$


were computed to all orders of perturbation theory in terms of a certain topological quantum field theory on the Riemann surface $\Sigma$. The total space $X$ is Calabi-Yau when the Chern class of the normal bundle to $\Sigma$ cancels the canonical class, i.e.

$$
\operatorname{deg}\left(L_{1}\right)+\operatorname{deg}\left(L_{2}\right)=-\chi(\Sigma)
$$

For example, when $\Sigma$ is a closed Riemann surface of genus $g$, we can take $\left(\operatorname{deg}\left(L_{1}\right), \operatorname{deg}\left(L_{2}\right)\right)=$ $(p+2 g-2,-p)$ for any integer $p$.

In [4] a Riemann surface $\Sigma$ is viewed as obtained by gluing of more basic building blocks: pants (P), annuli (A) and caps (C). The whole Calabi-Yau $X$ arises in this way from gluing simpler 3-folds which are rank 2 holomorphic bundles over Riemann surfaces with boundaries. Note that under gluing, the Euler characters of the base curves add, but moreover, the Chern classes of the normal bundles add.

Consider a Calabi-Yau $X$ involving a Riemann surface $\Sigma_{g, h}$ of genus $g$ with $h$ punctures or equivalently $h$ semi-infinite cylinders. The holomorphic maps from a worldsheet to $X$ are necessarily holomorphic maps to $\Sigma_{g, h}$. To get non-trivial such maps of finite area, we must add Lagrangian D-branes which circle the punctures (and that are 2-dimensional in the fiber direction) and consider holomorphic maps with boundaries on the D-branes. In this way, we can view cutting and pasting of the base Riemann surfaces and the corresponding Calabi-Yau's as either adding or cancelling off D-branes. The operations of gluing manifolds lead to composition of topological A-model amplitudes that satisfy all axioms of a two dimensional topological quantum field theory [⿴囗大 1 . In the course of this paper, we will explain from the physical standpoint what the TQFT of [4] is. As we will see, it is related to the large $N$ limit of the $q$-deformed Yang-Mills theory ( $q \mathrm{YM}$ ) on $\Sigma$.

By computing the open topological A-model amplitudes on a few Calabi-Yau manifolds, [4] get all others by gluing. The basic building blocks in [4] which we will need are the "Calabi-Yau caps" denoted by $C^{(-1,0)}$ and $C^{(0,-1)}$ carrying the first Chern classes $(-1,0)$ and $(0,-1)$ respectively and the pant $P^{(0,1)}$ with $(0,1)$ (see figure 1$)$.

The cap amplitude $C^{(-1,0)}$ is given by

$$
Z^{\mathrm{top}}\left(C^{(-1,0)}\right)=\sum_{R} d_{q}(R) q^{-k_{R} / 4} \operatorname{Tr}_{R} U
$$

Above,

$$
U=P e^{i \oint A}
$$


is the holonomy of the gauge field on the D-branes around the circle where D-brane meets $\Sigma$. We take the number of D-branes to be infinite, so $R$ labels a representation of $S U(\infty)$. The parameter $q$ is related to the string coupling constant $g_{s}$ by

$$
q=e^{-g_{s}} .
$$

The coefficient $d_{q}(R)$ is the quantum dimension of the symmetric group representation corresponding to the Young-Tableaux of $R$, defined by:

$$
d_{q}(R)=\prod_{\square \in R} \frac{1}{[h(\square)]_{q}},
$$

where the product runs over all the boxes in the Young tableaux of $R$ and $h$ is the hooklength of the corresponding box. The $q$-analogue $[x]_{q}$ of $x$ appearing in the formula is defined by

$$
[x]_{q}=q^{x / 2}-q^{-x / 2} .
$$

The exponent $k_{R}$ is given by

$$
k_{R}=2 \sum_{\square \in R}(i(\square)-j(\square))
$$

where $i, j(\square)$ label the location of the box in the tableaux. Note that $d_{q}(R) q^{k(R) / 4}$ is the same as the topological vertex amplitude $C_{R, 0,0}$ [5] with all but one representation set to be triviall:

$$
d_{q}(R) q^{k(R) / 4}=C_{R, 0,0}=W_{R 0} .
$$

This is a consistency check, since in this case we are considering the A-model corresponding to stack of D-branes on Calabi-Yau $X=\mathbf{C}^{3}$. This theory is considered in [0] and shown to compute $C_{R, 0,0}$.

Similarly, using the technology of 四, we can compute the Calabi-Yau "pant" $P^{(1,0)}$ (see fig. 1). This now carries 3 sets of representations corresponding to three stacks of D-branes at the 3 semi-infinite tubes with holonomies $U_{i}, i=1,2,3$ :

$$
Z^{\text {top }}\left(P^{(1,0)}\right)=\sum_{R} \frac{1}{d_{q}(R)} q^{k_{R} / 4} \operatorname{Tr}_{R} U_{1} \operatorname{Tr}_{R} U_{2} \operatorname{Tr}_{R} U_{3} .
$$

\footnotetext{
1 Our conventions differ here from [5] by $q \rightarrow q^{-1}$.
} 

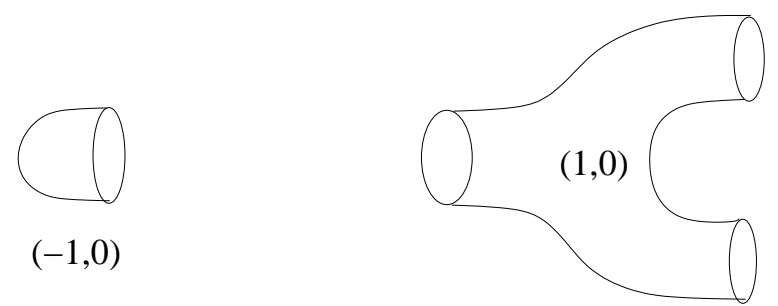

Fig.1 The Riemann surfaces corresponding to the cap amplitude $C^{(-1,0)}$ of the first Chern class $(-1,0)$ and pant $P^{(1,0)}$ with $(1,0)$.

These, together with the $C^{(0,-1)}$ cap and $P^{(0,1)}$ pant,

$$
\begin{aligned}
& Z^{\text {top }}\left(C^{(0,-1)}\right)=\sum_{R} d_{q}(R) q^{k_{R} / 4} \operatorname{Tr}_{R} U \\
& Z^{\text {top }}\left(P^{(0,1)}\right)=\sum_{R} \frac{1}{d_{q}(R)} q^{-k_{R} / 4} \operatorname{Tr}_{R} U_{1} \operatorname{Tr}_{R} U_{2} \operatorname{Tr}_{R} U_{3}
\end{aligned}
$$

suffice to compute any Calabi-Yau amplitude with or without D-branes by sewing.

Sewing $\Sigma_{L}$ and $\Sigma_{R}$ over their common boundary we get $\Sigma_{L \cup R}$. For this, the orientations of the corresponding boundary circles must be opposite. The operation of reversing the orientation of the boundary acts on the holonomy by $U \rightarrow U^{-1}$, and the corresponding path integrals are sewed together by

$$
Z^{\mathrm{top}}\left(\Sigma_{L \cup R}\right)=\int d U Z^{\mathrm{top}}\left(\Sigma_{L}\right)(U) Z^{\mathrm{top}}\left(\Sigma_{R}\right)\left(U^{-1}\right)
$$

In the representation basis

$$
Z^{\text {top }}(\Sigma)(U)=\sum_{R} Z_{R}^{\text {top }}(\Sigma) \operatorname{Tr}_{R}(U)
$$

because of the orthogonality of characters

$$
\int d U \operatorname{Tr}_{R_{1}} U \operatorname{Tr}_{R_{2}} U^{-1}=\delta_{R_{1} R_{2}}
$$

the sewing in (2.1) corresponds to identifying the left and the right representation $R$ and summing over $R$,

$$
Z^{\mathrm{top}}\left(\Sigma_{L \cup R}\right)=\sum_{P} Z^{\mathrm{top}}\left(\Sigma_{L}\right)_{P} Z^{\mathrm{top}}\left(\Sigma_{R}\right)_{P} .
$$


Note that, because the pant and the annulus are all diagonal in representations, the complex structure moduli of the Riemann surface one builds by cutting and pasting do not enter in the resulting topological string amplitudes. This is as it should be, since these correspond to the complex structure moduli of Calabi-Yau, and perturbative A-model topological string amplitudes better not depend on them.

For example, consider the A-model amplitude corresponding to a CalabiYau manifold fibered over a genus $g$ Riemann surface and bundle with the first Chern class $(2 g-2+p,-p)$. A quick counting shows that we need $(2 g-2)$ pants to get a closed genus $g$ Riemann surface, by thinking of it as composed of a necklace of $(g-1)$ handle adding operators. We can take $(2 g-2)$ pants of type $P^{(1,0)}$, and to get the bundle right, insert between them $p$ annuli $A^{(1,-1)}$ obtained by contracting $C^{(0,-1)}$ and $P^{(1,0)}$. This gives

$$
Z^{\mathrm{top}}\left(\Sigma_{g}\right)=\sum_{R}\left(\frac{1}{d_{q}(R)}\right)^{2 g-2} q^{(p+g-1) k_{R} / 2} e^{-t|R|}
$$

Note that in the above formula for $Z^{\text {top }}, d_{q}(R)$ is the $N \rightarrow \infty$ limit of the quantum dimension $\operatorname{dim}_{q}(R)$ of $U(N)$ representation with the same Youngtableaux. This is suggestive of the relation with Chern-Simons theory, which we will discuss in section 5 .

This answer captures all the non-trivial contributions to perturbative topological string amplitudes, but does not include contributions from constant maps. As is well known, these modify the amplitude by

$$
\begin{gathered}
Z^{\mathrm{top}}(q, t) \rightarrow Z_{0}(q, t) Z^{\mathrm{top}}(q, t), \\
Z_{0}(q, t)=M(q)^{\chi(X) / 2} \exp \left(a \frac{t^{3}}{6 g_{s}^{2}}+b \frac{t}{24}\right),
\end{gathered}
$$

where $M(q)=\prod_{n=1}^{\infty}\left(1-q^{n}\right)^{-n}$ is the McMahon function, $\chi(X)$ is the Euler characteristic, $a$ is the triple intersection of the Kähler class, and $b$ is related to $c_{2}$ of the Calabi-Yau $X$. In the non-compact model under discussion these are a little ambiguous, but we will find that the black hole counting agrees with the above if we take

$$
\chi(X)=2-2 g, \quad a=-\frac{1}{p(p+2 g-2)}, \quad b=\frac{p+2 g-2}{p},
$$


which we will adopt as our definition of perturbative topological string amplitude in this geometry. For simplicity of notation we will drop $Z_{0}(q, t)$ from the expressions below, but they should be included when comparing with the black hole prediction.

As another example, consider again a genus $g$ Riemann surface, but with $h$ punctures. This corresponds to insertion of $h$ additional pant diagrams. Choosing $(h-r)$ to be of type $P^{(1,0)}$ and $r$ of type $P^{(0,1)}$ we get a new Calabi-Yau manifold with fibers of degrees

$$
\left(\operatorname{deg}\left(L_{1}\right), \operatorname{deg}\left(L_{2}\right)\right)=\left(2 g-2+h+p^{\prime},-p^{\prime}\right)
$$

where $p^{\prime}=p-r$ and the topological string amplitude corresponding to it is

$$
Z^{\text {top }}\left(\Sigma_{g, h}\right)=\sum_{R}\left(\frac{1}{d_{q}(R)}\right)^{2 g-2+h} q^{\frac{\operatorname{deg}\left(L_{1}\right)-\operatorname{deg}\left(L_{2}\right)}{4} k_{R}} e^{-|R| t} \operatorname{Tr}_{R} U_{1} \cdots \operatorname{Tr}_{R} U_{h} \text {. }
$$

\subsection{D-branes in the Fiber}

So far we have considered Lagrangian D-branes wrapping 1-cycles on $\Sigma$, i.e. D-branes of topology $S^{1} \times C$ with the $S^{1}$ corresponding to a 1-cycle on $\Sigma$ and $C$ is the 2-dimensional subspace in the fiber. There is another class of Dbranes which will be relevant for us - these are Lagrangian D-branes wrapping 1-cycles in the fiber.

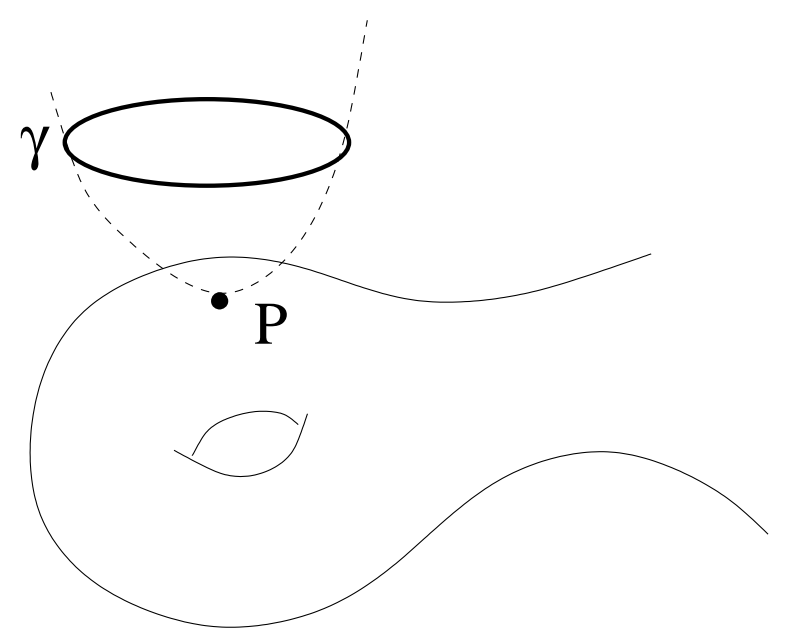

Fig.2 The figure depicts a D-bane wrapping Lagrangian cycle $\gamma$ in fiber over a point $P$ on the Riemann surface. Note that the D-brane need not touch the Riemann surface, rather there is a modulus corresponding to its position in the fiber. 
Consider as before $X=L_{1} \oplus L_{2} \rightarrow \Sigma$ for a fixed Riemann surface $\Sigma$. Pick a point $P$ on $\Sigma$, and let the D-brane lie in the fiber above this point. Let $z$ be a local coordinate centered at $P$. If $\left(u_{1}, u_{2}\right)$ are coordinates on the fiber $L_{1} \oplus L_{2}$ over $P$, then $\left(z, u_{1}, u_{2}\right)$ parameterizes a local $\mathbf{C}^{3}$ patch on $X$. In this patch there are Lagrangian D-branes of topology $C \times S^{1}$ where the $S^{1}$ corresponds to $\left|u_{1}\right|^{2}=$ const, for example. These are in fact the same D-branes considered in [5], and combining the results of [4] with that of [5], we can also obtain topological string amplitudes corresponding to them. The prescription is as follows.

We cut $\Sigma$ into two pieces, a cap $C_{P}$ containing $P$ and $\Sigma-C_{P}$. Correspondingly, cut $X$ into two pieces, one corresponding to $\mathbf{C}^{3}$ that fibers over $C_{P}$, the other to the fibration over $\Sigma-C_{P}$. We have

$$
Z(\Sigma)=\sum_{R} Z\left(C_{P}\right)_{R} Z\left(\Sigma-C_{P}\right)_{R}
$$

Now, adding a D-brane in fiber over $P$ on $\Sigma$, will replace $Z\left(C_{P}\right)_{R}$ by

$$
\sum_{Q} Z^{t o p}\left(C_{P}\right)_{R Q} \operatorname{Tr}_{Q} V
$$

where $V$ is the holonomy on this D-brane around the fiber $S^{1}$. Moreover, this should have a local effect that can be understood purely in the $\mathbf{C}^{3}$ patch we have cut out, and be independent of the rest of $X$. But, all the amplitudes with D-branes on $\mathbf{C}^{3}$ were computed in [5]. Adding a D-brane in the fiber must correspond, up to framing, to the topological vertex with one of the partitions trivial, i.e,

$$
Z^{t o p}\left(C_{P}\right)_{R Q}=W_{R Q}
$$

where $W_{R Q}(q)$ is the $N \rightarrow \infty$ limit of the $S$-matrix of WZW-model and ChernSimons theory

$$
W_{R Q}(q)=\lim _{N \rightarrow \infty} q^{\frac{N(|R|+|Q|)}{2}} \frac{S_{R Q}(q, N)}{S_{00}(q, N)}, \quad q=e^{-g_{s}} .
$$

For example, the amplitude on a genus $g$ Riemann surface with $h$ marked points and D-branes in the fiber over them is

$$
Z^{t o p}\left(\Sigma_{g, h}\right)=\sum_{R} \frac{W_{R R_{1}} \ldots W_{R, R_{h}}}{W_{R, 0}^{2 g-2+h}} q^{\frac{\operatorname{deg}\left(L_{1}\right)-\operatorname{deg}\left(L_{2}\right)}{4}} k_{R} \operatorname{Tr}_{R_{1}} V_{1} \cdots \operatorname{Tr}_{R_{h}} V_{h}
$$


where we used the fact that $W_{R, 0}=d_{R} q^{\frac{k_{R}}{4}}$. Note that D-branes need not be touching the Riemann surface, but they can be displaced some distance in the fiber. The modulus corresponding to where the D-brane is enters replacing

$$
\operatorname{Tr}_{R} V \rightarrow e^{-s|R|} \operatorname{Tr}_{R} V
$$

where $s$ is an arbitrary complex parameter. In this case it would correspond to the area of the disk that attaches the D-brane to the Riemann surface.

\subsection{An Alternative Derivation}

In this subsection we give a short alternative derivation of the topological string amplitudes in these backgrounds, analogous to the derivation of $2 \mathrm{~d}$ YangMills on a Riemann surface [6]. The idea is that by gluing rules all we need to determine are the cap, the annulus and the pant diagrams. The cap and the annulus diagram have been derived before [5] (as this is part of the standard topological vertex construction). For the pant diagram we would first argue that $Z^{\text {top }}(P)_{R_{1}, R_{2}, R_{3}}$ is zero unless all three representations are the same. As already noted the fact that the amplitudes of the A-model do not depend on the complex structure of the Riemann surface implies that $Z^{\text {top }}(P)_{R_{1}, R_{2}, R_{3}}$ vanishes unless all three representations have the same number of boxes. We can also argue that, more strongly, all three representations have to be the same for the vertex not to vanish. From the quantum foam picture [7] this is obvious because we cannot blow up the three different legs of the pant differently. We can only blow up all of the pant the same way, and this implies all representations are the same. Another way to show this, without using the quantum foam picture, is to consider a D-brane in the fiber above a point on the pant near one of the boundaries and study how the amplitude gets modified. Let us take the fiber D-brane representation to be $Q$.

First let us consider the topological amplitude for the annulus with representations $R_{1}, R_{2}$ on the annulus and $Q$ in the fiber $Z^{\text {top }}(A)_{R_{1}, R_{2}, Q}$. It is easy to see why this is zero unless $R_{1}=R_{2}$. If this were not to be the case, there should be a particular 'time' along the annulus (viewed as a cylinder) where the representation changes. The only way this can be is at the time corresponding to where the image of the D-brane is located. However we can move the image 
of the fiber D-brane on the annulus without changing the amplitude because this is just a complex structure deformation. We thus see that there is no point where $R_{1}$ can change to a different representation $R_{2}$. Therefore $R_{1}=R_{2}$. Now by putting a cap on one end we obtain

$$
Z^{\text {top }}(C)_{R} Z^{\text {top }}(A)_{R, R, Q}=W_{Q R}
$$

where the right side is obtained by noting that the new geometry is exactly what one has in the topological vertex. Thus we find, using $Z^{t o p}(C)_{R}=W_{0 R}$,

$$
Z^{\text {top }}(A)_{R_{1}, R_{2}, Q}=\delta_{R_{1}, R_{2}} \frac{W_{Q R_{1}}}{W_{0 R_{1}}}
$$

Armed with this result let us now consider the amplitude for the pant with three representations $R_{i}, i=1,2,3$ together with the fiber representation $Q$, $Z^{\text {top }}(P)_{R_{1}, R_{2}, R_{3}, Q}$. Again we exploit the crucial point that moving the projection of the fiber D-brane on the pant is a change in complex structure and does not affect the A-model amplitudes. Moving the fiber brane towards the $i$-th boundary and using the gluing rule and the above result for the annulus we see that

$$
Z^{\text {top }}(P)_{R_{1}, R_{2}, R_{3}, Q}=\frac{W_{Q R_{i}}}{W_{0 R_{i}}} Z^{t o p}(P)_{R_{1}, R_{2}, R_{3}}
$$

In other words, putting the fiber D-brane in representation $Q$ multiplies the amplitude by $W_{Q R_{i}} / W_{0 R_{i}}$ if we are near the $i$-th boundary of the pant. However, we can also move the fiber D-brane to the other boundaries of the pant without changing the amplitude. Thus $W_{Q R_{i}} / W_{0 R_{i}}$ should be the same for all $i$, and arbitrary $Q$ which implies that the $R_{i}$ are all equal. Thus all we have to find then is the representation dependence of the $Z^{t o p}(P)_{R, R, R}$. But this is easy, because gluing the cap should give the annulus, which implies $Z^{\text {top }}(C)_{R} Z^{\text {top }}(P)_{R, R, R}=1$ and leads to $Z^{\text {top }}(C)_{R, R, R}=1 / Z^{\text {top }}(C)_{R}=1 / W_{0 R}$. Here we have suppressed the framing dependence of the vertex which can be easily restored. 
3. Black Hole and Calabi-Yau $L_{1} \oplus L_{2} \rightarrow \Sigma_{g}$.

In this section we consider IIA string theory compactified on Calabi-Yau manifold $X$,

$$
X=L_{1} \oplus L_{2} \rightarrow \Sigma_{g},
$$

of the type studied in the previous section in the context of the topological string theory. We will take $\Sigma_{g}$ to be a genus $g$ Riemann surface, and

$$
\operatorname{deg}\left(L_{1}\right)=p+2 g-2, \quad \operatorname{deg}\left(L_{2}\right)=-p .
$$

We are interested in counting bound states of D4, D2 and D0 branes, where the D4-branes wrap

$$
C_{4}=L_{2} \rightarrow \Sigma_{g},
$$

and D2 branes wrap $\Sigma_{g}$. We fix the number of D4 branes to be $N$ and would like to count the ensemble of bound states on it. This can be done by studying the field theory on the brane and introducing certain interactions on it, which correspond to turning on chemical potentials for D2 and D0 branes.

The relevant quantum field theory has been studied in [2]. The case of interest in [2] was $\Sigma=T^{2}$ where the $\mathcal{N}=4$ topologically twisted gauge theory on $C_{4}$ was reduced to $2 \mathrm{~d}$ Yang-Mills gauge theory on $T^{2}$. The construction in [2] goes through for any genus Riemann surface, and we find that the theory reduces to a gauge theory on $\Sigma$, with one subtlety: The measure on field space in this reduction leads to a q-deformed 2d Yang-Mills theory on $\Sigma$. This measure does not affect the $T^{2}$ case studied in [2] and affects the partition function only for $g \neq 1$. We will therefore briefly review the construction in [2] and take into account the non-trivial measure factor for $g \neq 1$.

The world-volume gauge theory on the $\mathrm{N}$ D4-branes is the $\mathcal{N}=4$ topological $U(N)$ YM on $C_{4}$. Turning on chemical potentials for $D 0$ brane and $D 2$ brane correspond to introducing the observables

$$
S_{4 d}=\frac{1}{2 g_{s}} \int_{C_{4}} \operatorname{tr} F \wedge F+\frac{\theta}{g_{s}} \int_{C_{4}} \operatorname{tr} F \wedge K .
$$

where $K$ is the unit volume form of $\Sigma$. The parameters $g_{s}$ and $\theta$ are related to the chemical potentials $\phi^{0}$ and $\phi^{1}$ for $D 0$ and $D 2$ branes respectively as

$$
\varphi^{0}=\frac{4 \pi^{2}}{g_{s}}, \quad \varphi^{1}=\frac{2 \pi \theta}{g_{s}} .
$$


The topological theory is invariant under turning on a certain massive deformation which simplifies the theory. By using further deformation which correspond to a $U(1)$ rotation on the fiber, 目 the theory localizes to modes which are invariant under the $U(1)$ and effectively reduces the theory to a gauge theory on $\Sigma$. Let $z$ and $u$ be coordinates on $C_{4}$ corresponding to the Riemann surface and the fiber respectively, and let

$$
\Phi(z)=\int_{S_{z,|u|=\infty}^{1}} A .
$$

Here $S_{z,|u|=\infty}^{1}$ is the circle at infinity in the fiber over the point $z$ on $\Sigma$ and $A$ is the gauge field on the world-volume of the D4-branes, so the field $\Phi$ parameterizes the holonomy of the gauge field at infinity.

In reducing (3.1) to $2 \mathrm{~d}$ theory we should take into account two important effects. First, as shown in [2], the non-triviality of the fibration $L_{2} \rightarrow \Sigma$ generates the following term in the effective $2 \mathrm{~d}$ action:

$$
\delta S=-\frac{p}{2 g_{s}} \int_{\Sigma} \operatorname{tr} \Phi^{2}
$$

where $-p$ corresponds to the degree of $L_{2}$. The action becomes the action of $U(N)$ 2d YM theory

$$
S=\frac{1}{g_{s}} \int_{\Sigma} \operatorname{tr} \Phi \wedge F+\frac{\theta}{g_{s}} \int_{\Sigma} \operatorname{tr} \Phi \wedge K-\frac{p}{2 g_{s}} \int_{\Sigma} \operatorname{tr} \Phi^{2} .
$$

There is however an important subtlety: the field $\Phi$ comes from the holonomy of the gauge field at infinity, so it is periodic. More precisely, it is not $\Phi$ but $e^{i \Phi}$ which is a good variable. This does not affect the action, but it does affect the measure of the path integral. As a consequence, our theory is a certain deformation of $2 \mathrm{~d}$ YM, which can be naturally interpreted as a $q$-deformed version of the Yang-Mills theory as we shall see below. In the following we will provide two complementary ways to understand this theory. The first relies on localization and computes the path integral directly. The second uses an operatorial approach, where we cut $\Sigma$ into pieces on which we can solve the theory.

2 As discussed in [8] such equivariant deformations do not affect $\mathcal{N}=4$ Yang-Mills amplitudes, due to the high supersymmetry. 


\subsection{The q-Deformed 2d YM Theory I}

In this section we use topological invariance and localization to compute the path integral directly. We will begin by considering the ordinary $2 \mathrm{~d} \mathrm{YM}$, and then show how this gets modified in our case. We call our theory a $q$-deformed 2d YM, for the reason which will soon be apparent ${ }^{3}$.

Underlying the physical 2d YM theory at finite $p$ and $\theta$ is a topological YM theory [6] which is in fact the version of the $2 \mathrm{~d}$ YM theory arising naturally for us in the reduction of $\mathcal{N}=4$ topologically twisted Yang-Mills theory on $C_{4}$. To solve the $2 \mathrm{~d}$ YM theory it is convenient to use this topological formulation, as was done in [6] and [11] in two different approaches. We will briefly review both approaches below, only emphasizing how the change in measure for $\Phi$ affects the result. We first follow the approach in [1] and in the following subsection that of [6].

This topological YM theory has a BRST multiplet $(A, \psi, \phi)$ with the path integral

$$
\int \mathcal{D} A \mathcal{D} \phi \mathcal{D} \psi \exp \left[-\frac{1}{g_{s}} \int_{\Sigma} \operatorname{tr}(\phi F+\psi \wedge \psi)\right]
$$

and transformation laws

$$
\begin{aligned}
& \delta A=\epsilon \psi, \\
& \delta \psi=\epsilon D \phi, \\
& \delta \phi=0 .
\end{aligned}
$$

The field $\psi$ is an anti-commuting, Lie-algebra valued one form on $\Sigma$. Since it is appears quadratically in the action, it is usually neglected. The transformation laws imply that path-integral localizes on configurations with $\phi$ covariantly constant $D \phi=0$, where $\phi$ and $A$ can be simultaneously diagonalized. We will use this to find an Abelianization of the 2d YM, and then of our deformation of it. This has been done in [11], and we will sketch the main steps.

Write

$$
\phi=\phi_{i} T_{i}+\phi_{\alpha} T_{\alpha}, \quad A=A_{i} T_{i}+A_{\alpha} T_{\alpha}
$$

\footnotetext{
3 After completing this paper we became aware that q-deformed YM theory was studied previously in [9], [10].
} 
where $T_{i}$ are Cartan sub-algebra generators and $T_{\alpha}$ correspond to roots. We go to the gauge where $\phi$ is diagonal by setting $\phi_{\alpha}$ to zero. This gives Abelian $2 \mathrm{~d}$ YM with an additional coupling to off-diagonal modes of $A$ :

$$
\delta S_{0}=\int_{\Sigma} \sum_{\alpha} \alpha(\phi) A_{\alpha} A_{-\alpha} .
$$

Moreover, to fix the gauge properly, we must also take care of the path integral measure. This is done by introducing a pair of ghosts $\left(c_{\alpha}, \bar{c}_{\alpha}\right)$ with coupling

$$
\delta S_{1}=\int_{\Sigma} \sum_{\alpha} \alpha(\phi) \bar{c}_{-\alpha} c_{\alpha} .
$$

Integrating over the $c$ 's and the $A$ 's gives a ratio of one-loop determinants of a complex scalar of fermionic statistics and commuting one-form which is hermitian. The non-zero modes cancel between them [11], and the zero modes give:

$$
\frac{\Delta(\phi)^{2 b_{0}(\Sigma)}}{\Delta(\phi)^{b_{1}(\Sigma)}}=\Delta(\phi)^{\chi(\Sigma)}
$$

where

$$
\Delta(\phi)=\prod_{1 \leq i<j \leq N}\left(\phi_{i}-\phi_{j}\right)
$$

Note that for $g>1$ this appears singular at places where $\Delta_{H}(\phi)$ vanishes. When $\Delta(\phi)$ vanishes the gauge group becomes non-Abelian, so we have integrated out a massless field, and the singularity is a consequence of it. In fact [1] the singular points give no contribution to the path integral. By giving a bare mass term to $A_{\alpha}$ we can regularize the path integral $\sum_{\alpha>0} \mu A_{\alpha} A_{-\alpha}$ it is easy to see that the problematic points give a vanishing contribution for any non-zero value of $\mu$, and by continuity for $\mu$ zero as well.

It is now simple to turn $p$ and $\theta$ back on, to the physical theory, viewing it as inserting a $Q$-closed (but not exact) observable to the path integral of the topological theory. Now, exactly the same discussion would go through in our case where $\phi$ is compact, however we have to make sure that the terms we add in $\delta S$ respect this. The easiest way to do this is by the method of images, more precisely by adding an infinite sequence of multiplets $\left(c_{\vec{n}}, \bar{c}_{\vec{n}}\right)$, which couple to $\vec{\phi}+2 \pi \vec{n}$. The effect of this is to replace $\Delta(\phi)$ by

$$
\Delta_{H}(\phi)=\prod_{1 \leq i<j \leq N} 2 \sin \left(\frac{\phi_{i}-\phi_{j}}{2}\right) .
$$


To summarize, we end up with an Abelian gauge theory:

$$
Z^{q Y M}(\Sigma)=\frac{1}{N !} \int^{\prime} \prod_{i} d \phi_{i}\left[\Delta_{H}(\phi)\right]^{\chi(\Sigma)} \exp \left[\int_{\Sigma} \frac{p}{2 g_{s}} \sum_{i} \phi_{i}^{2}-\frac{\theta}{g_{s}} \sum_{i} \phi_{i}-\frac{1}{g_{s}} \sum_{i} F_{i} \phi_{i}\right]
$$

where $\int^{\prime} \prod_{i} d \phi_{i}$ denotes the path integral with points with $\Delta_{H}(\phi)=0$ omitted. The $\frac{1}{N !}$ factor corresponds to dividing by the volume of the Weyl group, as a discrete permutation symmetry inherited from $U(N)$. The normalization of the path integral has ambiguities coming in part from the choice of regularization. As explained in [6], for the theory at hand, which is essentially topological or more precisely invariant under area preserving diffeomorphisms of $\Sigma$, the different regularizations differ by additions of terms of the form

$$
a \int_{\Sigma} R+b \int_{\Sigma} K=a \chi(\Sigma)+b
$$

to the action. For us, the constants $a$ and $b$ will be fixed by the black hole physics. In the following, we will not worry about the normalizations until the end of the subsection.

Note that above coincides, at $p=0=\theta$ with the partition function of Chern-Simons theory on $S^{1} \times \Sigma$ derived in [11] by a different, but related method. At more general values of $p$, this should correspond to the ChernSimons theoryt on $S^{1}$ bundle over $\Sigma$ with the first Chern class $-p$. It should be clear why the Chern-Simons theory appears: The non-compact four-cycle $C_{4}$ wrapped by the $\mathrm{D} 4$ branes has this as a boundary. The action (3.1) should be viewed as providing a definition of the Chern-Simons theory for non-integer values of $k$ in $g_{s}=2 \pi /(k+N)$, taking $g_{s}$ to be in principle arbitrary. We will discuss the relation of the D4 brane theory on $X$ to the topological string theory on $X$ in section 5 .

We can in fact give a completely explicit expression for the above path integral. Integrating over the gauge fields reduces [11] to a sum over nontrivial $U(1)^{N}$ bundles on $\Sigma$. These are classified by their first Chern classes,

$$
F_{i}=2 \pi r_{i} K, \quad r_{i} \in \mathbf{Z}
$$

4 This theory has been recently studied in 12 with a different motivation. 
Summing over all flux configurations, we find that integral over $\phi_{i}$ gets contribution from

$$
\phi_{i}=i g_{s} n_{i}, \quad n_{i} \in \mathbf{Z}
$$

so that partition function is given by

$$
Z^{q Y M}(\Sigma)=\frac{1}{N !} \sum_{n_{i} \in \mathbf{Z}}^{\prime}\left(\prod_{1 \leq i<j \leq N}\left[n_{i}-n_{j}\right]_{q}\right)^{2-2 g} q^{\frac{p}{2} C_{2}(\vec{n})} e^{i \theta C_{1}(\vec{n})}
$$

where

$$
\begin{gathered}
q=e^{-g_{s}}, \\
C_{2}(\vec{n})=\sum_{i=1}^{N} n_{i}^{2}, \quad C_{1}(\vec{n})=\sum_{i=1}^{N} n_{i},
\end{gathered}
$$

and $[x]_{q}$ is the $q$-analogue of $x$ defined as usual by $[x]_{q}=q^{\frac{x}{2}}-q^{-\frac{x}{2}}$. We must recall that points where $\Delta_{H}(\phi)=0$ are omitted from the path integral, and correspondingly we omit terms in the sum where $n_{i}=n_{j}$. We used $\sum_{n_{i} \in Z}^{\prime}$ to denote this modified sum.

In fact, we can rewrite the above as a sum over representations $\mathcal{R}$ of $U(N)$. By using the Weyl invariance to restrict the fundamental Weyl chamber, $n_{1}>$ $n_{2}>\cdots>n_{N} \geq 0$, and then letting $n_{i}=\mathcal{R}_{i}$ where $\mathcal{R}_{i}$ 's label the lengths of rows of $\mathcal{R}$. Finally it is convenient to shift the $\mathcal{R}_{i}$ by $\rho_{i}=\frac{1}{2}(N-2 i+1)$, i.e. we shift by the Weyl vector 6 Then, $C_{1,2}$ become the corresponding Casimir's of $U(N)$

$$
\begin{aligned}
& C_{2}(\mathcal{R})=k_{\mathcal{R}}+N|\mathcal{R}|, \quad k_{\mathcal{R}}:=\sum_{i=1}^{N} \mathcal{R}_{i}\left(\mathcal{R}_{i}-2 i+1\right), \\
& C_{1}(\mathcal{R})=|\mathcal{R}|, \quad|\mathcal{R}|:=\sum_{i=1}^{N} \mathcal{R}_{i},
\end{aligned}
$$

and the partition sum is expressed as

$$
Z^{q Y M}(\Sigma)=\sum_{\mathcal{R}} S_{0 \mathcal{R}}^{2-2 g} q^{\frac{p}{2} C_{2}(\mathcal{R})} e^{i \theta C_{1}(\mathcal{R})}
$$

5 Note that the sign of theta is not meaningful, the theories at $\theta$ and $-\theta$ are equivalent.

6 More precisely, this is valid only when $N$ is odd, otherwise we shift $n_{j}=\mathcal{R}_{j}+\frac{N}{2}-j$ and also introduce a shift of $\theta$ angle. 
where $S_{0 \mathcal{R}}=S_{00} \operatorname{dim}_{q}(\mathcal{R})$ and the quantum dimension of representation $\mathcal{R}$ is given by

$$
\operatorname{dim}_{q}(\mathcal{R})=\prod_{1 \leq i<j \leq N} \frac{\left[\mathcal{R}_{i}-\mathcal{R}_{j}+j-i\right]_{q}}{[j-i]_{q}}
$$

(note that $S_{00}$ is the denominator in the above expression). The reader should note here that we have deliberately distinguished the representations $R$ of $S U(N)$ from representations $\mathcal{R}$ of $U(N)$ here. This somewhat technical point will be of crucial importance in section 5 where we consider the large $N$ limit of the $q$-deformed YM theory.

One can recognize in the above the building blocks of the $U(N)$ ChernSimons theory:

$$
\operatorname{dim}_{q}(\mathcal{R})=S_{\mathcal{R} 0} / S_{00}
$$

where $S_{\mathcal{R} \mathcal{Q}}$ correspond to entries of the S-matrix of $U(N)_{k} W Z W$ model, albeit at non-integer value of level $k$. In fact $k$ here is a pure imaginary number. In particular we do not have any truncation of the representations, which is usually associated to integer $k$.

We finish this subsection by giving the normalized $q$-deformed YM partition function. As we mentioned above, the normalization is ambiguous due terms of the form (3.9), but fixed for us by black hole physics to be discussed in section 5 to bel

$$
Z^{q Y M}(\Sigma)=\alpha\left(g_{s}, \theta\right) \sum_{\mathcal{R}} S_{0 \mathcal{R}}^{2-2 g} q^{\frac{p}{2} C_{2}(\mathcal{R})} e^{i \theta C_{1}(\mathcal{R})}
$$

where

$$
\alpha\left(g_{s}, \theta\right)=q^{\frac{\rho^{2}(p+2 g-2)}{2 p}} e^{\frac{N \theta^{2}}{2 p g_{s}}} q^{(2-2 g)\left(\rho^{2}+\frac{N}{24}\right)} .
$$

\subsection{The q-Deformed 2d YM Theory II}

We now give an operatorial approach for computing the partition function whose advantage is that it will make a similarity to the topological theory of section 2 apparent. We will follow closely the approach of [6]: we first solve the theory on a sphere with three holes, and then get the rest by sewing.

To begin with note that, just as in the case of ordinary $2 \mathrm{~d} Y \mathrm{YM}, \Phi$ and $A$ are canonically conjugate variables. In quantizing the theory on a cylinder,

\footnotetext{
7 See (5.9)-(5.14) in section 5.
} 
we can take as basis of the Hilbert space to be gauge invariant functions of $A$. These are given by $\chi_{\mathcal{R}}(U)=\operatorname{Tr}_{\mathcal{R}} U$ where

$$
U=P \exp i \oint A
$$

On these $\Phi(x)$ acts as $\Phi(x)=g_{s} \frac{\partial}{\partial A(x)}$ or

$$
\Phi_{a}(x) \chi_{\mathcal{R}}(U)=i \chi_{\mathcal{R}}\left(T_{a} U\right)
$$

where $T_{a}$ are generators of the lie algebra $\Phi(x)=\sum_{a} \Phi_{a}(x) T_{a}$. Recall that, since we are studying a $U(N)$ gauge theory, $U$ is unitary and $\mathcal{R}$ labels representation of $U(N)$.

We will first solve the theory in the topological limit, obtained by setting $p=0$ and then study the consequences of turning on $p$. For simplicity, we will also turn off the theta angle and restore it back later.

Consider the path integral on a pant $P$ i.e. a sphere with three punctures with holonomies $U_{i}, i=1,2,3$ around them. This is of the form

$$
Z(P)\left(U_{1}, U_{2}, U_{3}\right)=\sum_{\mathcal{R}_{1}, \mathcal{R}_{2}, \mathcal{R}_{3}} Z(P)_{\mathcal{R}_{1}, \mathcal{R}_{2}, \mathcal{R}_{3}} \operatorname{Tr}_{\mathcal{R}_{1}} U_{1} \operatorname{Tr}_{\mathcal{R}_{2}} U_{2} \operatorname{Tr}_{\mathcal{R}_{3}} U_{3}
$$

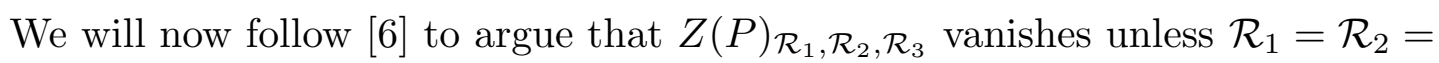
$\mathcal{R}_{3}=\mathcal{R}$. Inserting an arbitrary operator $\mathcal{O}(\Phi(x))$ at any one of the punctures picks out the corresponding Casimir of the representation at that puncture. Using the invariance of the observable on the point of insertion, we can move the operator $\mathcal{O}(\Phi)$ to any other puncture.

By similar argument, the path integral on an annulus $A$ of length $T$ is given by

$$
Z(A)\left(U_{1}, U_{2}\right)=\sum_{\mathcal{R}} \operatorname{Tr}_{\mathcal{R}} U_{1} \operatorname{Tr}_{\mathcal{R}} U_{2}
$$

As annulus amplitude is the propagator, we would usually have representation $\mathcal{R}$ weighted by $e^{-H(\mathcal{R}) T}$, where $H$ is the Hamiltonian and $T$ is the length of the propagator. However $H$ vanishes in the topological theory: at $p=0=\theta$.

Finally, consider the path integral on a cap $C$

$$
Z(C)(U)=\sum_{\mathcal{R}} Z(C)_{\mathcal{R}} \operatorname{Tr}_{\mathcal{R}} U
$$


First, note that the cap and the pant amplitudes are not independent. Gluing the cap $C$ to the pant $P$ we must recover the annulus amplitude:

$$
\int d U Z(C)\left(U^{-1}\right) Z(P)\left(U, U_{1}, U_{2}\right)=Z(A)\left(U_{1}, U_{2}\right)
$$

This implies

$$
Z(C)_{\mathcal{R}}=\frac{1}{Z(P)_{\mathcal{R}}}
$$

where we used a $U(N)$ analogue of the $S U(N)$ orthogonality relation (2.2)

$$
\int d U \operatorname{Tr}_{\mathcal{R}} U \operatorname{Tr}_{\mathcal{Q}} U^{-1}=\delta_{\mathcal{R}, \mathcal{Q}}
$$

Now we come to the main point of the discussion, which is the computation of the cap amplitude of the deformed $2 \mathrm{~d}$ YM.

To begin with, recall what happens in ordinary the 2d YM. Computing a path integral with fixed holonomy $U$ on the boundary simply gives a delta function on the holonomy $\delta(U-1)=\sum_{\mathcal{R}} \operatorname{dim}(\mathcal{R}) \operatorname{Tr}_{\mathcal{R}}(U)$, so that

$$
Z^{2 \mathrm{dYM}}(U)(C)=\sum_{\mathcal{R}} \operatorname{dim}(\mathcal{R}) \operatorname{Tr}_{\mathcal{R}} U
$$

Now, we could have also computed this in the basis where we fix $\Phi(x)$ on the boundary and not $U$. Since $\Phi(x)$ and $A$ are canonically conjugate, this is related to the above by a simple Fourier transform,

$$
Z^{2 \mathrm{dYM}}(C)(\Phi)=\int D U e^{\frac{1}{g_{s}} \oint_{\partial C} \operatorname{Tr} \Phi A} Z^{2 \mathrm{dYM}}(C)(U)
$$

where the path integral is taken on the boundary of the disk. We will show in the appendix A that the integral is simply given by

$$
Z^{2 \mathrm{dYM}}(C)(\phi)=\prod_{1 \leq i<j \leq N}\left(\phi_{j}-\phi_{i}\right)=\Delta(\phi)
$$

where $\phi_{i}(i=1, \ldots N)$ are eigenvalues of $\Phi$. Note that $\Delta(\phi)$ is the same function (3.7) we met in the previous subsection.

Now, let us return to the deformed YM theory. In this case $\phi_{i}$ 's are periodic, so the wave function (3.14) is not well defined. One can make it well defined by 
adding images under $\phi_{i} \rightarrow \phi_{i}+2 \pi$. This, as in the previous subsection, replaces $\Delta(\phi)=\prod_{1 \leq i<j \leq N}\left(\phi_{j}-\phi_{i}\right)$ with

$$
\Delta_{H}(\phi)=\prod_{1 \leq i<j \leq N} 2 \sin \left(\frac{\phi_{i}-\phi_{j}}{2}\right)
$$

Alternatively, we can express the wave function in terms of the holonomy basis, by undoing the Fourier transform. This gives:

$$
Z^{q Y M}(C)(U)=\sum_{\mathcal{R}} \operatorname{dim}_{q}(\mathcal{R}) \operatorname{Tr}_{\mathcal{R}} U
$$

where the quantum dimension of representation $\mathcal{R}$ is given by (3.11). Note that $\operatorname{dim}_{q}(\mathcal{R})$ becomes $\operatorname{dim}(\mathcal{R})$ in the limit $g_{s} \rightarrow 0$.

Finally, to get back to the physical theory by turning on $p$ and $\theta$. As explained in [6], the difference between the physical and the topological theory is only that the Hamiltonian of the former is not vanishing, and it is given by 8

$$
H=\frac{1}{2} g_{s} p C_{2}-i \theta C_{1}
$$

This gives the area dependence to the amplitudes. For example, the amplitude for an annulus of length $T=1$ is

$$
Z^{q Y M}(A)\left(U_{1}, U_{2}\right)=\sum_{\mathcal{R}} q^{p C_{2}(\mathcal{R}) / 2} e^{i \theta \mathcal{R}} \operatorname{Tr}_{\mathcal{R}} U_{1} \operatorname{Tr}_{\mathcal{R}} U_{2}
$$

Since we can change the area of the pant $P$ and the cap $C$ by adding annuli, the corresponding amplitudes of the physical theory are:

$$
Z^{q Y M}(P)\left(U_{1}, U_{2}, U_{3}\right)=f \sum_{\mathcal{R}} \frac{1}{\operatorname{dim}_{q}(\mathcal{R})} q^{p C_{2}(\mathcal{R}) / 2} e^{i \theta \mathcal{R}} \operatorname{Tr}_{\mathcal{R}} U_{1} \operatorname{Tr}_{\mathcal{R}} U_{2} \operatorname{Tr}_{\mathcal{R}} U_{3}
$$

and

$$
Z^{q Y M}(C)(U)=\frac{1}{f} \sum_{\mathcal{R}} \operatorname{dim}_{q}(\mathcal{R}) q^{p C_{2}(\mathcal{R}) / 2} e^{i \theta \mathcal{R}} \operatorname{Tr}_{\mathcal{R}} U
$$

where we have normalized the area of the resulting surface to one. The normalization factor $f$, which is independent of the $U_{i}$ 's, cannot be fixed by this argument and depends on the normalization of the path-integral. Consistency

8 See footnote 4 , on page 15. 
with the previous discussion leads to $f=1 / S_{00}$. From these we can get arbitrary amplitudes of our deformed 2d YM theory on any Riemann surface by sewing.

For example, we can finally compute the partition function on a genus $g$ Riemann surface. We do so by sewing $(2 g-2)$ pant amplitudes and find

$$
Z^{q Y M}\left(\Sigma_{g}\right)=S_{00}^{2-2 g} \sum_{\mathcal{R}}\left(\frac{1}{\operatorname{dim}_{q}(\mathcal{R})}\right)^{2 g-2} q^{p C_{2}(\mathcal{R}) / 2} e^{i \theta \mathcal{R}}
$$

where we have set the area of the surface to 1 (we also need the prefactor $\alpha\left(g_{s}, \theta\right)$, discussed before, in comparing with black-hole physics). Note that this amplitude, at $p=0=\theta$, is identical to that of the Chern-Simons theory on $\Sigma_{g} \times S^{1}$. For general $p$ but $\theta=0$ this is equivalent to the result for the ChernSimons theory on a circle bundle over $\Sigma$ where the circle bundle is identified with the circles of fixed norm of the $L_{2}$ bundle over $\Sigma$. The only difference being that here $k$ is not an integer and we do not have the usual truncation of representations which occurs for integer $k$.

Finally, in the above, we have mainly been considering wave-functions in the polarization where we specify $A$ on the boundary. We can rewrite all the amplitudes corresponding to manifolds with boundaries in terms of keeping $\Phi=-i g_{s} \frac{\partial}{\partial A}$ fixed on the boundary. For example, consider the pant amplitude.

$$
Z^{q Y M}(P)\left(V_{1}, V_{2}, V_{3}\right)=\sum_{\mathcal{R}_{1}, \mathcal{R}_{2}, \mathcal{R}_{3}} Z^{V}(P)_{\mathcal{R}_{1}, \mathcal{R}_{2}, \mathcal{R}_{3}} \operatorname{Tr}_{\mathcal{R}_{1}} V_{1} \operatorname{Tr}_{\mathcal{R}_{2}} V_{2} \operatorname{Tr}_{\mathcal{R}_{3}} V_{3}
$$

where, since only $e^{i \Phi}$ is well defined,

$$
V_{i}=e^{i \Phi_{i}} \quad(i=1,2,3)
$$

Now, viewed from the three-dimensional perspective, we have a three-manifold with three $T^{2}$ torus boundaries. Fixing $V=e^{i \Phi}$ on the boundary, fixes the holonomy around $a$-cycle of the $T^{2}$ say (corresponding to the $S^{1}$ fiber over the pant $P$ ), whereas fixing $U=P e^{i \oint A}$ fixes holonomy around the $b$ cycle. Now we've derived the amplitude corresponding to fixing $U$. We could compute from it the amplitude with $V$ fixed if we could exchange the $a$ and the $b$ cycle. 
Fortunately, in the Chern-Simon theory, such operation is well known and is implemented by the $S$ matrix

$$
S_{\mathcal{R}_{1} \mathcal{R}_{2}}
$$

of the $W Z W$ model. So we have:

$$
Z_{\mathcal{R}_{1}, \mathcal{R}_{2}, \mathcal{R}_{3}}^{V}=\sum_{\mathcal{R}} \frac{S_{\mathcal{R}_{1} \mathcal{R}} S_{\mathcal{R}_{2} \mathcal{R}} S_{\mathcal{R}_{3} \mathcal{R}}}{S_{0 \mathcal{R}}} q^{p C_{2}(\mathcal{R}) / 2} e^{i \theta C_{1}(\mathcal{R})} .
$$

The right hand side, at $p=0=\theta$ is simply the Verlinde formula, for non-integer $k$. This of course is not a surprise: in that case, we are effectively computing a Chern-Simons amplitude on $S^{1} \times S^{2}$ with Wilson-lines on $S^{1}$ in representation $\mathcal{R}_{1}, \mathcal{R}_{2}, \mathcal{R}_{3}$ over three points in the $S^{2}$, and the results are the fusion coefficients, analytically continued to arbitrary $k$ using the Verlinde formula [13].

\section{Black Hole Entropy}

In this section we will count the number of BPS states of the black hole that arises from $N$ D4-branes on $C_{4}$ with any number of D0-branes and D2-branes wrapping $\Sigma$. As we will note below, in order to do this we have to perform a modular transformation on the partition function evaluated in the previous section.

In the topologically twisted $\mathcal{N}=4$ YM theory discussed in the last section we turned on observable corresponding to D-brane charges

$$
S_{4 d}=\frac{1}{2 g_{s}} \int_{C_{4}} \operatorname{tr} F \wedge F+\frac{\theta}{g_{s}} \int_{C_{4}} \operatorname{tr} F \wedge K,
$$

where $K$ is Kähler class of $\Sigma_{g}$. The $D_{0}$ and $D_{2}$ brane charges $q_{0}, q_{1}$ are measured by

$$
q_{0}=\frac{1}{8 \pi^{2}} \int_{C_{4}} \operatorname{tr} F \wedge F, \quad q_{1}=\frac{1}{2 \pi} \int_{C_{4}} \operatorname{tr} F \wedge K .
$$

It was shown in [14 that the functional integral

$$
Z^{q \mathrm{YM}}=\int \mathcal{D} A \exp \left(-\frac{1}{2 g_{s}} \int_{C_{4}} \operatorname{tr} F \wedge F-\frac{\theta}{g_{s}} \int_{C_{4}} \operatorname{tr} F \wedge K\right)
$$

with an appropriate gauge fixing has an expansion of the form,

$$
Z^{q \mathrm{YM}}=\sum_{q_{0}, q_{1}} \Omega\left(q_{0}, q_{1} ; N\right) \exp \left[-\frac{4 \pi^{2}}{g_{s}} q_{0}-\frac{2 \pi \theta}{g_{s}} q_{1}\right],
$$


where $\Omega\left(q_{0}, q_{1} ; N\right)$ is the Euler characteristic of the moduli space of $U(N)$ instantons in the topological sector set by (4.1) and it can also be regarded as the Witten index for the black hole with the given D-brane charges. By setting

$$
\varphi^{0}=\frac{4 \pi^{2}}{g_{s}}, \quad \varphi^{1}=\frac{2 \pi \theta}{g_{s}}
$$

the instanton expansion of $Z^{q \mathrm{YM}}$ can be expressed as

$$
Z^{q \mathrm{YM}}=\sum_{q_{0}, q_{1}} \Omega\left(q_{0}, q_{1} ; N\right) \exp \left[-q_{0} \varphi^{0}-q_{1} \varphi^{1}\right]
$$

For the non-compact manifold $C_{4}$, the charges $q_{0}, q_{1}$ can be fractional. We will find below that $q_{0} \in \frac{1}{2 p} \mathbf{Z}$ and $q_{1} \in \frac{1}{p} \mathbf{Z}$.

The expressions we gave for $Z^{q \mathrm{YM}}$ in the previous section, however, are expansions in $q=e^{-g_{s}}$ as in $(3.10) 9$

$$
\begin{aligned}
Z^{q \mathrm{YM}}\left(\Sigma_{g}\right)= & \alpha\left(g_{s}, \theta\right) q^{-\frac{p \rho^{2}}{2}} \\
& \times \frac{1}{N !} \sum_{\vec{n} \in \mathbf{Z}^{N}}^{\prime}\left(\prod_{i<j}\left[n_{j}-n_{i}\right]_{q}\right)^{2-2 g} q^{\frac{p}{2} \vec{n} \cdot \vec{n}} e^{i \theta \vec{n} \cdot \vec{e}},
\end{aligned}
$$

where $\sum_{\vec{n}}^{\prime}$ the modified sum omitting $n_{i}=n_{j}$ for $i \neq j, \alpha\left(g_{s}, \theta\right)$ is defined in (3.13), and $\vec{e}=(1,1, \ldots, 1)$. Fortunately, the beautiful fact that $\mathcal{N}=4 \mathrm{YM}$ theory has $S$-duality saves the day. The $S$-duality implies that $Z^{q \mathrm{YM}}\left(g_{s}, N\right)$ is a modular form, which turns the expression (4.4) into the form (4.3). We will now demonstrate this.

Let us first review the genus $g=1$ case studied in [2]. For $g=1$, the partition sum can be expressed in terms of elliptic functions [15] as in

$Z^{q \mathrm{YM}}\left(T^{2}\right)=\frac{q^{\frac{N \theta^{2}}{2 p g_{s}}+\frac{(1-p)}{2} \rho^{2}}}{N !}\left[\vartheta(z, \tau)^{N}-\frac{N !}{2 !(N-2) !} \vartheta(z, \tau)^{N-2} \vartheta(2 z, 2 \tau)-\cdots\right]$

where $\vartheta(z, \tau)=\sum_{n \in Z} e^{\pi i \tau n^{2}+2 i \pi z n}$ and

$$
z=\frac{\theta}{2 \pi}, \quad \tau=\frac{i}{2 \pi} p g_{s}
$$

9 We have chosen the normalization of the $q \mathrm{YM}$ path integral to include the prefactor $\alpha\left(g_{s}, \theta\right) q^{-\frac{p \rho^{2}}{2}}$. This choice is required for the factorization into chiral blocks in section 5 . 
Note that the first term $\vartheta(\zeta, \tau)^{N}$ in the right-hand side of (4.5) is obtained from (4.4) by ignoring the constraints $n_{i} \neq n_{j}$ for $i \neq j$, and the other terms $\vartheta(z, \tau)^{N-2} \vartheta(2 z, 2 \tau)+\cdots$ are corrections to incorporate these constraints. Applying the modular transformation,

$$
\vartheta(z, \tau)=\frac{1}{\sqrt{-i \tau}} e^{-i \pi \frac{z^{2}}{\tau}} \vartheta\left(\frac{z}{\tau},-\frac{1}{\tau}\right)
$$

to the first term (4.5), we find

$$
Z^{q \mathrm{YM}}\left(T^{2}\right)=\frac{q^{\frac{(1-p)}{2} \rho^{2}}}{N !}\left(\frac{2 \pi}{p g_{s}}\right)^{\frac{N}{2}} \sum_{\vec{m} \in \mathbf{Z}} e^{-\frac{1}{2 p} \vec{m}^{2} \varphi^{0}+\frac{1}{p} \vec{m} \cdot \vec{e} \varphi^{1}}+\cdots
$$

The other terms can be transformed similarly. Comparing this with (4.7), we find that $q_{0}, q_{2}$ are fractions $q_{0} \in \frac{1}{2 p} \mathbf{Z}, q_{1} \in \frac{1}{p} \mathbf{Z}$ and that the Witten index of black hole states is given by

$$
\Omega\left(q_{0}, q_{1} ; N\right)=\#\left|\left\{\vec{m} \in \mathbf{Z}^{N} \mid q_{0}=\frac{1}{2 p} \vec{m}^{2}, q_{1}=\frac{1}{p} \vec{e} \cdot \vec{m}\right\}\right|+\cdots
$$

For $g=0$, things are simpler since the factor $\prod_{i<j}\left[n_{i}-n_{j}\right]_{q}^{2}$ automatically takes into account the constraints $n_{i} \neq n_{j}(i \neq j)$ and we may extend summation over unrestricted $n_{i} \in Z$ without any subtractions. It is useful to expand this factor as a sum over the Weyl group $W$ of $U(N)$ as in

$$
\prod_{1 \leq i<j \leq N}\left[n_{i}-n_{j}\right]_{q}=\sum_{w \in W} \epsilon(w) q^{-w(\rho) \cdot n}
$$

where $\epsilon(w)= \pm 1$ is the parity of the Weyl group element $w$. We can then express the partition sum as

$$
Z^{q \mathrm{YM}}\left(S^{2}\right)=\frac{q^{\frac{N \theta^{2}}{2 p g_{s}}+\frac{N}{12}-\frac{\left(p^{2}-5 p+2\right)}{2 p} \rho^{2}}}{N !} \sum_{w, w^{\prime} \in W} \epsilon(w) \epsilon\left(w^{\prime}\right) \prod_{k=1}^{N} \vartheta\left(z_{k}\left(w, w^{\prime}\right), \tau\right)
$$

where

$$
\tau=\frac{i}{2 \pi} p g_{s}, \quad z_{k}\left(w, w^{\prime}\right)=\frac{1}{2 \pi}\left(\theta-i g_{s} a_{k}\left(w, w^{\prime}\right)\right)
$$

and

$$
a_{k}\left(w, w^{\prime}\right)=w(\rho)_{k}+w^{\prime}(\rho)_{k}
$$


Now we use the modular transformation (4.6) of $\vartheta(z, \tau)$ to recast $Z^{q \mathrm{YM}}\left(S^{2}\right)$ in the form:

$$
\begin{aligned}
Z^{q \mathrm{YM}}\left(S^{2}\right)= & \frac{q^{\frac{N}{12}-\frac{\left(p^{2}-5 p+2\right)}{2 p} \rho^{2}}}{N !}\left(\frac{2 \pi}{p g_{s}}\right)^{N / 2} \sum_{w, w^{\prime} \in W} \epsilon(w) \epsilon\left(w^{\prime}\right) \\
& \times \sum_{\vec{n} \in \mathbf{Z}^{N}} \exp \left[-\frac{2 \pi^{2}}{p g_{s}}\left(\vec{n}+\frac{i g_{s}}{2 \pi} \vec{a}\left(w, w^{\prime}\right)\right)^{2}+\frac{2 \pi \theta}{p g_{s}} \vec{n} \cdot \vec{e}\right] .
\end{aligned}
$$

It is instructive to compare this with mathematical results on the Euler characteristic of the moduli space of instantons on the four-manifold $C_{4}=$ $\mathcal{O}(-p) \rightarrow \mathbf{P}^{1}$.

When $p=1$, the partition function (4.11) can be expressed as

$$
Z^{q \mathrm{YM}}=f\left(\varphi^{0}\right)\left[\sum_{n} e^{-\frac{1}{2} n^{2} \varphi^{0}-n \varphi^{1}}\right]^{N}
$$

for some $\varphi^{0}$ dependent factor $f\left(\varphi^{0}\right)$. The prefactor would be related to the bound state of the D0 brane to the D4 brane which is ambiguous in the present context due to the non-compactness of the D4 brane. (In the compact case it would have been $\eta^{-N \chi\left(C_{4}\right)}\left(\varphi_{0}\right)$.) However the $D 2$ branes bound to D0 and D4 branes are unambiguous because they are frozen on the compact part of the geometry, which is the Riemann surface. In this case, $C_{4}$ is the total space of $\mathcal{O}(-1)$ over $\mathbf{P}^{1}$, which is a blowup of $\mathbf{C}^{2}$ at one point, and (4.12) exactly reproduces the $\varphi^{1}$ dependence of the blow-up formula conjectured in [14] and proven in [16].

When $p=2$, 4.11 becomes

$$
\begin{aligned}
& Z^{q \mathrm{YM}}=\frac{q^{\frac{\rho^{2}+N}{12}}}{2 N !}\left(\frac{2 \pi}{p g_{s}}\right)^{N / 2} \sum_{w, w^{\prime} \in W} \epsilon(w) \epsilon\left(w^{\prime}\right) q^{-\frac{1}{2 p} a\left(w, w^{\prime}\right)^{2}} \\
& \times \sum_{\vec{n} \in \mathbf{Z}^{N}}(-1)^{\vec{n} \cdot \vec{a}\left(w, w^{\prime}\right)} e^{-\frac{1}{4} \vec{n}^{2} \varphi^{0}-\frac{1}{2} \vec{e} \cdot \vec{n} \varphi^{1}} \\
&=\frac{q^{\frac{\rho^{2}+N}{12}}}{2 N !}\left(\frac{2 \pi}{p g_{s}}\right)^{N / 2} \sum_{w, w^{\prime} \in W} \epsilon(w) \epsilon\left(w^{\prime}\right) q^{\frac{1}{4 \pi p} a\left(w, w^{\prime}\right)^{2}} \\
& \times \prod_{i=1}^{N}\left[\sum_{r=0, \frac{1}{2}}(-1)^{2 r a_{i}\left(w, w^{\prime}\right)} \sum_{n \in \mathbf{Z}} e^{-(n+r)^{2} \varphi^{0}-(n+r) \varphi^{1}}\right] .
\end{aligned}
$$


Note that the characters of the level $1 S U(2)$ affine Lie algebra are given by

$$
\chi_{r}^{\text {level } 1}\left(\varphi^{0}, \varphi^{1}\right)=\frac{\sum_{n} e^{-(n+r)^{2} \varphi^{0}-(n+r) \varphi^{1}}}{\prod_{n=1}^{\infty}\left(1-e^{-n \varphi^{0}}\right)}
$$

where $r=0, \frac{1}{2}$ corresponds to the spin 0 and $\frac{1}{2}$ representations respectively. Since (4.13) shows that the $\varphi^{1}$-dependence of $Z^{q \mathrm{YM}}$ is given by a product of $N$ of such characters, one can expanded $Z^{q \mathrm{YM}}$ as a sum over the characters of the $S U(2)$ affine Lie algebra of level $N$ with $\varphi^{0}$ dependent coefficients. This agrees with the result by Nakajima [17] that the level $N$ affine algebra acts on the cohomologies of the moduli space of $U(N)$ instantons on $\mathcal{O}(-2) \rightarrow \mathbf{P}^{1}$, which is our $C_{4}$ in the case of $p=2$. The choice of the $S U(2)$ representation is determined by the boundary condition at the infinity. Since the $S$-duality transformation mixes up the boundary conditions, it is reasonable that $Z^{q \mathrm{YM}}$ computed in the previous section becomes a sum of the affine $S U(2)$ characters after the $S$-dual transformation.

Thus, we have demonstrated for $g=0$ and 1 that our computation of $Z^{q \mathrm{YM}}$ based on the reduction to the $q$-deformed $2 d \mathrm{YM}$ on $\Sigma_{g}$ agrees with the instanton expansion of the $\mathcal{N}=4 \mathrm{YM}$ on $C_{4}$ and that $Z^{q \mathrm{YM}}$ is indeed the generating function of the BPS black hole that arises from wrapping D4branes on $C_{4}$. It would be interesting to test this for $g \geq 2$ also. Our next task, however, is to relate $Z^{q \mathrm{YM}}$ to closed topological string amplitudes on the Calabi-Yau manifold $X$.

\section{Large $N$ Limit of $Z^{q \mathrm{YM}}$ and the Relation to Perturbative Topolog- ical Strings}

The deep relation, conjectured in [1], between 4-dimensional black holes and topological strings predicts that the partition function of black holes on $X$ $Z_{B H}=Z^{q \mathrm{YM}}$, for large charges, is related to the perturbative topological string partition function $Z^{\text {top }}$ on $X$ as

$$
Z^{q \mathrm{YM}} \sim Z^{\mathrm{top}} \bar{Z}^{\mathrm{top}}
$$

In this section we will aim to get a better understanding of this relation by considering the large $N$ (i.e. large black hole charge) limit of the quantum $2 d$ YM theory on a Riemann surface $\Sigma$ of genus $g$. 
The conjecture of [1] predicts a precise relation of the parameters of the YM theory describing the black hole and the dual gravity, or topological string theory. According to the conjecture [1], when one relates the black hole to the topological string theory, the moduli of the Calabi-Yau manifold are fixed by the black hole attractor mechanism. This fixes the real parts of the projective coordinates $\left(X^{0}, X^{1}\right)$ on Calabi-Yau moduli space to magnetic charges of D6 and D4 branes and their imaginary parts are the chemical potentials $\varphi^{0}, \varphi^{1}$ for the electric D0 and D2 brane charges. In the current setup, there is no D6 brane magnetic charge, and we have $N$ D4 branes. Now, the magnetic charge for a single D4 brane, if we measure in terms of electric units of D2 branes wrapping $\Sigma$, is given by the intersection number of $\Sigma$ and the 4 -cycle the D4 brane wraps. In the present case this is

$$
\#\left(\Sigma \cap C_{4}\right)=p+2 g-2
$$

as can easily be seen by deforming $\Sigma$ away from $C_{4}=L_{2} \rightarrow \Sigma$ using a generic section of $L_{1}$. Thus, in the black hole background, the projective moduli for the closed topological string are fixed to be

$$
X^{0}=i \frac{\varphi^{0}}{\pi}, \quad X^{1}=(p+2 g-2) N-i \frac{\varphi^{1}}{\pi} .
$$

In the previous section, we saw that the chemical potentials are related to the $q \mathrm{YM}$ parameters as $\varphi^{0}=\frac{4 \pi^{2}}{g_{s}}$, and $\varphi^{1}=\frac{2 \pi \theta}{g_{s}}$, so that

$$
X^{0}=\frac{4 \pi i}{g_{s}}, \quad X^{1}=(p+2 g-2) N-2 i \frac{\theta}{g_{s}} .
$$

Since the Kähler modulus $t$ corresponding to the base $\Sigma$ of the Calabi-Yau manifold is given by

$$
t=2 \pi i \frac{X^{1}}{X^{0}}
$$

in terms of projective coordinates, we expect that the topological closed string theory which is the gravity dual of the $U(N) 2 \mathrm{~d} q \mathrm{YM}$ theory has $t$ fixed to be

$$
t=\frac{1}{2}(p+2 g-2) N g_{s}-i \theta
$$


The large $N$ limit of the ordinary 2d YM theory, a cousin of our theory, 10 was studied in [3]. They find that, at large $N$, the Hilbert space of the YM theory factorizes as

$$
\mathcal{H}_{\mathrm{YM}} \rightarrow \mathcal{H}_{\text {chiral }} \otimes \mathcal{H}_{\text {antichiral }}
$$

with $\mathcal{H}_{\text {chiral }}$ corresponding to representations $\mathcal{R}_{+}$with much less than $N$ boxes, and $\mathcal{H}_{\text {antichiral }}$ corresponding to representations $\mathcal{R}_{-}$of order $N$ boxes. The same will apply to the $q$-deformed YM theory. Corresponding to this, the partition function $Z^{q \mathrm{YM}}$ should factorize as

$$
Z^{q \mathrm{YM}} \sim Z_{q \mathrm{YM}}^{+} Z_{q \mathrm{YM}}^{-}
$$

It is natural to expect that the two factorizations (5.1) and (5.4) are related. More precisely, one would expect that chiral $q \mathrm{YM}$ partition function $Z_{q \mathrm{YM}}^{+}$can be written as a holomorphic function of $t$, and identified with the topological string amplitude $Z^{\text {top }}(t)$

$$
Z_{q \mathrm{YM}}^{+}\left(N, \theta, g_{s}\right) \sim Z^{\mathrm{top}}\left(t, g_{s}\right) .
$$

We will show that this is indeed the case in the class of Calabi-Yau manifolds at hand, with some important subtlety that we will describe in detail below.

The cases $X=L_{1} \oplus L_{2} \rightarrow \Sigma_{g}$ for $g \geq 1$ and $g=0$ work somewhat differently in their technical aspects, so we will consider them separately below.

\subsection{Factorization into Chiral Blocks for Genus $g>1$ Case.}

We consider the large $N$ limit of the BH partition function (3.12) for $g>1$

$$
Z^{q \mathrm{YM}}\left(\Sigma_{g}\right)=\alpha\left(g_{s}, \theta\right) \sum_{\mathcal{R}} S_{0 \mathcal{R}}^{2-2 g} q^{\frac{p}{2} C_{2}(\mathcal{R})} e^{i \theta C_{1}(\mathcal{R})} .
$$

As we will show below in this section the natural value of the normalization constant $\alpha\left(g_{s}, \theta\right)$ is as defined in (3.13). Here $\mathcal{R}$ labels $U(N)$ representations. It will be more convenient use the decomposition of $\mathcal{R}$ in terms of $S U(N)$ representation $R$ and the $U(1)$ charge $m$. First, we will have to recall how various quantities pertaining to $U(N)$ relate to those of $S U(N)$ and the $U(1)$.

10 Since $q=e^{-g_{s}}$ and $g_{s} \sim g_{\mathrm{YM}}^{2}$, the ordinary $2 \mathrm{~d}$ YM theory with finite $g_{\mathrm{YM}}$ is not a limit of our $q$-deformed theory. 
The Young-tableaux of $\mathcal{R}$ differs from that of $R$ by having $r$ columns of length $N$ attached:

$$
\begin{aligned}
& \mathcal{R}_{i}=R_{i}+r, \quad i=1, \ldots, N-1 \\
& \mathcal{R}_{N}=r .
\end{aligned}
$$

The $U(1)$ charge is then given by

$$
m=|R|+N r, \quad r \in \mathbf{Z} .
$$

The $U(N)$ Casimir's are related to the $S U(N)$ Casimir's as

$$
C_{1}(\mathcal{R})=m, \quad C_{2}(\mathcal{R})=C_{2}(R)+\frac{m^{2}}{N}
$$

where

$$
C_{2}(R)=k_{R}+N|R|-\frac{|R|^{2}}{N}, \quad k_{R}=\sum_{i=1}^{N-1} R_{i}\left(R_{i}-2 i+1\right)
$$

Their quantum dimensions are equal

$$
\operatorname{dim}_{q}(\mathcal{R})=\operatorname{dim}_{q}(R)=\prod_{1 \leq i<j \leq N} \frac{\left[R_{i}-R_{j}+j-i\right]_{q}}{[j-i]_{q}} .
$$

Just as for ordinary $2 d \mathrm{YM}$ [3], the factorization of the Hilbert space at large $N$ is captured by writing an irreducible $S U(N)$ representation $R$ in terms of the coupled representations $R_{+} \bar{R}_{-}$with $R_{+}$and $R_{-}$labelling states in $\mathcal{H}_{\text {chiral }}$ and $\mathcal{H}_{\text {antichiral }}$, respectively. The Casimir's decompose as follows: the $U(1)$ charge $m$ of $R$ becomes

$$
m=N l+\left|R_{+}\right|-\left|R_{-}\right|,
$$

where $l=r+R_{-, 1}$, and

$$
C_{2}\left(R_{+} \bar{R}_{-}\right)=C_{2}\left(R_{+}\right)+C_{2}\left(R_{-}\right)+2 \frac{\left|R_{+}\right|\left|R_{-}\right|}{N} .
$$




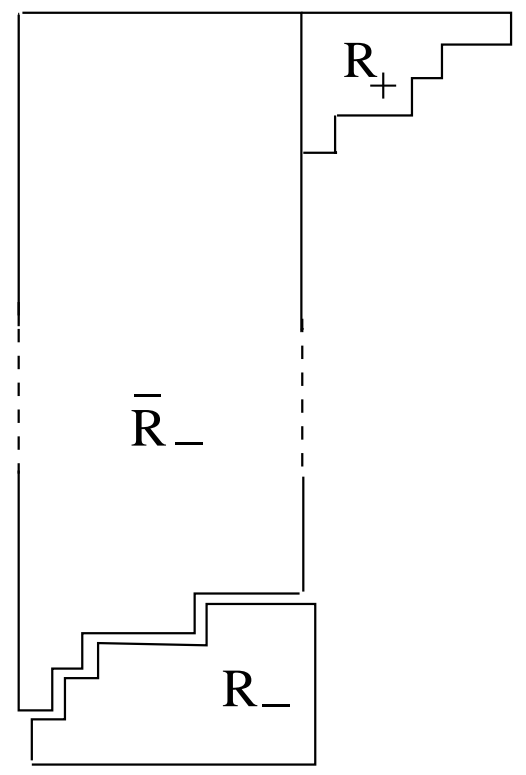

Fig.4 The figure depicts an $S U(N)$ representation $R$ as a coupled representation $R_{+} \bar{R}_{-}$. The representation $\bar{R}_{-}$is conjugate to representation $R_{-}$.

Using this, and trading $N$ and $\theta$ for $t$ and $\bar{t}$ defined by (5.3), the $q \mathrm{YM}$ partition function becomes

$$
\begin{gathered}
Z^{q \mathrm{YM}}\left(\Sigma_{g>1}\right)=\alpha\left(g_{s}, \theta\right) \sum_{l \in \mathbf{Z}} \sum_{R_{+}, R_{-}}\left(S_{00} \operatorname{dim}_{q} R_{+} \bar{R}_{-}\right)^{2-2 g} q^{\frac{p}{2}\left(k_{R_{+}}+k_{R_{-}}\right)} e^{i \theta\left(\left|R_{+}\right|-\left|R_{-}\right|\right)} \\
\times q^{\frac{p N}{2}\left(\left|R_{+}\right|+\left|R_{-}\right|\right)} e^{-\frac{\left(t^{2}-\bar{t}^{2}\right) l}{2(p+2 g-2) g_{s}}} e^{-\frac{p(t+\bar{t}) l^{2}}{2(p-2+2 g)}} e^{-p g_{s} l\left(\left|R_{+}\right|-\left|R_{-}\right|\right)}
\end{gathered}
$$

So far, we have merely rewritten (5.9) and now we are ready to turn to its factorization at large $N$. The key fact, shown in Appendix B, is the following relation

$$
\begin{aligned}
q^{\rho^{2}+\frac{N}{24}} S_{00} \operatorname{dim}_{q}\left(R_{+} \bar{R}_{-}\right)= & \frac{M(q) \eta^{N}(q)}{K_{R_{+} R_{-}}(Q, q)} W_{R_{+}}^{2}(q) W_{R_{-}}^{2}(q) \\
& \times(-)^{\left|R_{+}\right|+\left|R_{-}\right|} q^{-\frac{1}{2}\left(k_{R_{+}}+k_{R_{-}}\right)} Q^{-\frac{1}{2}\left(\left|R_{+}\right|+\mid R_{-}\right)}
\end{aligned}
$$

where $M(q)$ is MacMahon function, $\eta(q)=q^{\frac{1}{24}} \prod_{j=1}^{\infty}\left(1-q^{j}\right)$ and

$$
K_{R_{+} R_{-}}(Q, q):=\sum_{P} Q^{|P|} W_{P R_{+}}(q) W_{P R_{-}}(q)
$$

Moreover, the normalization factor $\alpha\left(g_{s}, \theta\right)$, defined in (3.13), can be written as

$$
\alpha\left(g_{s}, \theta\right)=\left|\Upsilon\left(t, g_{s}\right)\right|^{2}\left(q^{\rho^{2}+\frac{N}{24}}\right)^{2-2 g}
$$


where

$$
\Upsilon\left(t, g_{s}\right)=\exp \left(-\frac{t^{3}}{6 p(p+2 g-2) g_{s}^{2}}+\frac{(p+2 g-2) t}{24 p}\right) .
$$

Using (5.10) and (5.12) we can recast $Z_{g>1}^{q \mathrm{YM}}$ in the large $N$ limit as a sum of "chiral blocks":

$Z^{q \mathrm{YM}}\left(\Sigma_{g>1}\right)=\sum_{l \in \mathbf{Z}} \sum_{R_{1}, \ldots, R_{2 g-2}} Z_{R_{1}, \ldots, R_{2 g-2}}^{q \mathrm{YM},+}\left(t+p g_{s} l\right) Z_{R_{1}, \ldots, R_{2 g-2}}^{q \mathrm{YM},+}\left(\bar{t}-p g_{s} l\right)$.

The chiral block $Z_{R_{1}, \ldots, R_{2 g-2}}^{q \mathrm{YM},+}$ is defined by

$$
\begin{aligned}
Z_{R_{1}, \ldots, R_{2 g-2}}^{q \mathrm{YM},+}(t) & =Z_{0}\left(t, g_{s}\right) \eta^{\frac{t \chi}{(p-\chi) g_{s}}} e^{-\frac{t\left(\left|R_{1}\right|+\ldots\left|R_{2 g-2}\right|\right)}{(p-2+2 g)}} \\
& \times \sum_{R} q^{\frac{1}{2}(p+2 g-2) k_{R}} e^{-t|R|} \frac{W_{R_{1} R}(q) \ldots W_{R_{2 g-2} R}(q)}{W_{0 R}(q)^{4 g-4}}
\end{aligned}
$$

which agrees, as we will discuss below, with the perturbative topological string amplitudes with $(2 g-2)$ stacks of D-branes inserted in the fiber. The extra prefactor $\eta^{\frac{t \chi}{(p-\chi) g_{s}}}$ needs to be explained. However this factor has only a genus 0 contribution perturbatively. Namely, by using the modular property of the Dedekind eta function, we find that this factor contributes as

$$
\eta^{\frac{t \chi}{(p-\chi) g_{s}}} \sim \exp \left(-\frac{c t}{g_{s}^{2}}\right)+(\text { non }- \text { perturbative })
$$

where $c=\frac{\pi^{2} \chi}{6(p-\chi)}$. It is reassuring that this can be viewed as a correction to the topological string amplitudes at genus zero. This is possible because the power of $t$ is less than three and the fact that topological string partition function at genus 0 is ambiguous up to the addition of a quadratic polynomial in $t$. Thus we can redefine the degree 0 contributions (2.5) to be

$$
\widehat{Z}_{0}\left(t, g_{s}\right)=Z_{0}\left(t, g_{s}\right) \exp \left(-\frac{c t}{g_{s}^{2}}\right) \text {. }
$$

We will come back to the interpretation of the blocks in the topological string context, after we have discussed the genus zero case. 


\subsection{Factorization into Chiral Blocks for Genus $g=0$ Case.}

To find the large $N$ limit of the $q$-deformed YM partition function on a genus zero Riemann surface $Z^{q \mathrm{YM}}\left(S^{2}\right)$ we proceed in a similar way, except that we write the quantum dimension of the coupled representation $R_{+} \bar{R}_{-}$slightly differently: (see Appendix B)

$$
\begin{aligned}
q^{\rho^{2}+\frac{N}{24}} S_{00} \operatorname{dim}_{q}\left(R_{+} \bar{R}_{-}\right)= & M(q) \eta^{N}(q) N_{R_{+} R_{-}}(Q, q)(-)^{\left|R_{+}\right|+\left|R_{-}\right|} \\
& \times q^{-\frac{k_{R_{+}+k_{R_{-}}}}{2}} Q^{-\frac{\left|R_{+}\right|+\left|R_{-}\right|}{2}}
\end{aligned}
$$

where $q=e^{-g_{s}}, Q=e^{-g_{s} N}$ as before, and

$$
N_{R_{+} R_{-}}(Q, q):=\sum_{P}(-)^{|P|} Q^{|P|} W_{P R_{+}}(q) W_{P^{T} R_{-}}(q) .
$$

Using (5.17) and (5.18) as well as (5.8), we find the large $N$ limit of $Z^{q \mathrm{YM}}\left(S^{2}\right)$ given by (3.10) is

$$
Z^{q \mathrm{YM}}\left(S^{2}\right)=\sum_{l \in \mathbf{Z}} \sum_{R_{1}, R_{2}} Z_{R_{1}, R_{2}}^{q \mathrm{YM},+}\left(t+p g_{s} l\right) Z_{R_{1}, R_{2}}^{q \mathrm{YM},-}\left(\bar{t}-p g_{s} l\right),
$$

where the chiral block, $Z_{R_{1}, R_{2}}^{q \mathrm{YM},+}(t)$, is defined by

$$
\begin{aligned}
Z_{R_{1}, R_{2}}^{\mathrm{YYM},+}(t)=\widehat{Z}_{0}\left(t, g_{s}\right) & q^{\frac{k_{R_{1}}+k_{R_{2}}}{2}} e^{-\frac{t\left(\left|R_{1}\right|+\left|R_{2}\right|\right)}{(p-2)}} \\
& \times \sum_{R} q^{\frac{(p-2) k_{R}}{2}} e^{-t|R|} W_{R_{1} R}(q) W_{R R_{2}^{T}}(q) .
\end{aligned}
$$

and the 'anti-chiral block', unlike the higher genus case, is transposed:

$$
Z_{R_{1}, R_{2}}^{q \mathrm{YM},-}(\bar{t})=Z_{R_{1}^{T}, R_{2}^{T}}^{+}(\bar{t})(-)^{\left(\left|R_{1}\right|+\left|R_{2}\right|\right)}
$$

\subsection{Large $N$ Expansion and Perturbative Topological String}

To summarize the results of the previous two subsections, we have found that in the large $N$ expansion the partition function of the $q$-deformed YM theory corresponding to $N$ D4-branes on the zero section of $L_{1}$ in

$$
X_{\Sigma}=L_{1} \oplus L_{2} \rightarrow \Sigma
$$


factorizes as

$$
Z^{q \mathrm{YM}}(\Sigma)=\sum_{l \in \mathbf{Z}} \sum_{R_{1}, \ldots, R_{|2 g-2|}} Z_{R_{1}, \ldots R_{|2 g-2|}}^{q \mathrm{YM},+}\left(t+l p g_{s}\right) Z_{R_{1}, \ldots R_{|2 g-2|}}^{q \mathrm{YM},+}\left(\bar{t}-l p g_{s}\right)
$$

where $p$ is related to the degree of $L_{1}$ by $\operatorname{deg}\left(L_{1}\right)=-p$. What is the interpretation of the chiral block in terms of the topological string theory on $X$ ?

For concreteness, let us focus on $g \geq 1$ case. According to (5.15), the chiral blocks are expressed as

$$
Z_{R_{1}, \ldots, R_{|2 g-2|} \mathrm{YM},+}(t)=\widehat{Z}_{0} e^{-\frac{t\left(\left|R_{1}\right|+\ldots\left|R_{2 g-2}\right|\right)}{(p-2+2 g)}} \sum_{R} \frac{W_{R_{1} R}(q) \ldots W_{R_{2 g-2} R}(q)}{W_{0 R}(q)^{4 g-4}} q^{\frac{1}{2}(p+2 g-2) k_{R}} e^{-t|R|}
$$

where $\widehat{Z}_{0}$ contains the classical pieces (5.16).

First notice that in the large $N$ expansion of the $q$-deformed YM partition function, there is a limit where the anti-chiral theory decouples, namely taking $\bar{t} \rightarrow \infty$, at fixed $t$. Here we are treating $t$ and $\bar{t}$ as independent variables. In this limit only the trivial representation contributes in the sum over $R_{1}, \ldots, R_{2 g-2}$ in $(5.14)$ because of the factors $e^{-\frac{\bar{t}\left(\left|R_{1}\right|+\ldots\left|R_{2 g-2}\right|\right)}{(p-2+2 g)}}$ in $Z^{q \mathrm{YM}-}$. Thus, we find

$$
\lim _{\bar{t} \rightarrow \infty} Z^{q \mathrm{YM}}(t, \bar{t})=Z_{0, \ldots, 0}^{q \mathrm{YM},+}(t)
$$

up to a trivial anti-chiral piece. A look back at section 2 shows that this is exactly equal to the perturbative closed topological string partition function (2.3) for the same Calabi-Yau:

$$
Z_{0, \ldots, 0}^{q \mathrm{YM},+}(t)=Z^{\mathrm{top}}\left(X_{\Sigma_{g}}\right)(t)
$$

Thus, in the limit where the anti-chiral part of the $q \mathrm{YM}$ theory decouples, we recover the perturbative topological string theory amplitude on $X$, at the value of Kähler parameter fixed by the attractor mechanism.

However, this is clearly not all, and $\left|Z^{\text {top }}\left(X_{\Sigma_{g}}\right)(t)\right|^{2}$ is but the first in the sum over chiral blocks in the large $N$ expansion of $q \mathrm{YM}$ on $\Sigma_{g}$. Amazingly, the other chiral blocks also have an interpretation in terms of theory on $X_{g}$, but now involving D-branes! Another look back at section 2 shows that the object which appears in the higher chiral blocks is also topological string amplitude on $X$, but with with $(2 g-2)$ D-branes in the fiber over $(2 g-2)$ points on $\Sigma$ 
given in equation (2.6), with the degrees of the line bundles adjusted to the Calabi-Yau at hand, i.e. $\operatorname{deg}\left(L_{1}\right)=2 g-2+p, \operatorname{deg}\left(L_{2}\right)=-p$. Moreover, the D-branes are moved off the Riemann surface by an amount $t /(p+2 g-2)$. With this understanding, we have the identity:

$$
Z_{R_{1}, \ldots, R_{|2 g-2|}}^{q \mathrm{YM},+}(t)=Z_{R_{1}, \ldots, R_{|2 g-2|}}^{\mathrm{top}}(t)
$$

It should be clear from the preceding discussion that $Z_{R_{1}, \ldots, R_{|2 g-2|}}^{\text {top }}(t)$ can itself be obtained as a $\bar{t} \rightarrow \infty$ limit of the $q \mathrm{YM}$ amplitude - this time one obtained by gluing $(4 g-4)$ "dual" pant vertices (3.15) to get a Riemann surface of genus $g$ with $(2 g-2)$ punctures [18].

To understand the geometric meaning of the chiral blocks, it is useful to express the large $N$-expansion of $Z^{q \mathrm{YM}}$ in terms of an integral over $(2 g-2)$ holonomies $V_{1}, \cdots, V_{2 g-2} \in U(\infty)$ as

$$
\begin{aligned}
Z^{q \mathrm{YM}}\left(\Sigma_{g>1}\right)=\sum_{l \in \mathbf{Z}} \int d V_{1} \cdots d V_{2 g-2} & Z^{\mathrm{top}}\left(g_{s}, t+p g_{s} l ; V_{1}, \ldots, V_{2 g-2}\right) \\
& \times Z^{\mathrm{top}}\left(g_{s}, \bar{t}-p g_{s} l ; V_{1}^{-1}, \ldots, V_{2 g-2}^{-1}\right)
\end{aligned}
$$

where

$$
Z^{\text {top }}\left(g_{s}, t ; V_{1}, \ldots, V_{2 g-2}\right)=\sum_{R_{1}, \ldots, R_{2 g-2}} Z_{R_{1}, \ldots, R_{2 g-2}}^{\text {top }} \operatorname{Tr}_{R_{1}} V_{1} \ldots \operatorname{Tr}_{R_{2 g-2}} V_{2 g-2}
$$

and we used the orthogonality of the characters (2.2). Each of the D-branes on the fiber described in section 2.1 intersects with $C_{4}$ at a non-contractible circle on its worldvolume. Thus, one can regard $V_{i}(i=1, \ldots, 2 g-2)$ as a holonomy of the gauge field on the $i$-th stack of D-branes around the cycle, keeping track of the way the worldsheet ends on the D-branes. We take the number of Dbranes at each stack to be infinite so that the representations $R_{1}, \ldots, R_{2 g-2}$ can be arbitrary.

These D-branes are directly related to the presence of $\Omega$-points in the large $N$ limit of the ordinary 2d YM theory [3]. To see the connection, it is useful to move to a more geometric basis for the chiral blocks. We can do this by using the Frobenius formula, which expresses the trace $\operatorname{Tr}_{R}(U)$ for any representation $R$ 
in terms of a sum of products of traces of $U$ in the fundamental representations as

$$
\operatorname{Tr}_{R}(U)=\frac{1}{n !} \sum_{\sigma \in S_{n}} \chi_{R}(\sigma) \operatorname{tr}_{\vec{k}(\sigma)} V
$$

where

$$
\operatorname{tr}_{\vec{k}(\sigma)} V=\prod_{i=1}\left(\operatorname{tr} V^{i}\right)^{k_{i}(\sigma)}
$$

The sum in (5.23) is over elements of symmetric group $S_{n}$ of $n$ elements, where $n=|R|$ is the number of boxes in the Young diagram corresponding to the representation $R, \chi_{R}(\sigma)$ is the character of the representation of $S_{n}$ corresponding to the same Young diagram, tr is the trace over the fundamental representation, and $k_{i}(\sigma)$ is the number of cycles of length $i$ in the permutation $\sigma \in S_{n}$. Note that $\operatorname{tr}_{\vec{k}(\sigma)} V$ depends only on the conjugacy class of the permutation $\sigma$. By using the orthogonality relations $(2.2)$ it follows from (5.23),

$$
\int d V \operatorname{tr}_{\vec{k}} V \operatorname{tr}_{\vec{k}^{\prime}} V^{-1}=\delta\left(\vec{k}, \vec{k}^{\prime}\right) \zeta(\vec{k})
$$

where

$$
\zeta(\vec{k})=\prod_{i=1}^{\infty} i^{k_{i}^{a}} k_{i}^{a} !
$$

One can re-express $(5.22)$ as

$$
Z^{q \mathrm{YM}}\left(\Sigma_{g}\right)=\sum_{l \in \mathbf{Z}_{\vec{k}_{1}, \ldots, \vec{k}_{2 g-2}}} \sum_{\vec{k}^{1}, \ldots, \vec{k}^{2 g-2}}^{\mathrm{top}}\left(g_{s}, t+p g l\right) Z_{\vec{k}^{1}, \ldots, \vec{k}^{2 g-2}}^{\mathrm{top}}\left(g_{s}, \bar{t}-p g l\right) \prod_{a=1}^{2 g-2} \zeta\left(\vec{k}^{a}\right)
$$

where $Z_{\vec{k}^{1}, \ldots \vec{k}^{2 g-2}}^{\text {top }}$ is defined by

$$
Z^{\text {top }}\left(g_{s}, t ; V_{1}, \ldots, V_{2 g-2}\right)=\sum_{\vec{k}^{1}, \ldots, \vec{k}^{2 g-2}} Z_{\vec{k}^{1}, \ldots \vec{k}^{2 g-2}}^{\mathrm{top}}\left(g_{s}, t\right) \operatorname{tr}_{\vec{k}^{1}} V_{1} \cdots \operatorname{tr}_{\vec{k}^{2 g-2}} V_{2 g-2}
$$

By construction, $Z_{\vec{k}^{1}, \ldots, \vec{k}^{2 g-2}}^{\text {top }}\left(g_{s}, t\right)$ is the topological string amplitude with the constraint that the worldsheet ends on the $a$-th stack of D-branes with $k_{i}^{a}$ boundaries wrapping on the non-contractible cycle $i$-times $(i=1,2, \ldots)$. The expression (5.24) suggests that the complete topological string partition function includes gluing of the holomorphic and anti-holomorphic topological string amplitudes together so that the boundaries of the holomorphic and the 
anti-holomorphic worldsheets match up on the D-branes. Note that the combinatorial factor $\prod_{a=1}^{2 g-2} \zeta\left(\vec{k}^{a}\right)$ is exactly the number of ways the boundaries can be glued together. Since $Z^{\text {top }}$ is an exponential of a sum of connected worldsheets, the full topological string partition function involves arbitrary numbers of connected worldsheets, holomorphic and anti-holomorphic. Thus, despite the appearance of D-branes, worldsheets contributing to the topological string amplitudes are still closed, except that they are piecewisely holomorphic or anti-holomorphic.

In this way, the $2 g-2$ stacks of D-branes generate analogues of the $2 g-2 \Omega$ points that appear in the large $N$ limit of the ordinary $2 d \mathrm{YM}$ [3]. To complete the story, one would need to explain why exactly $2 g-2$ D-branes are involved in the non-perturbative completion of the string theory on $L_{1} \oplus L_{2} \rightarrow \Sigma_{g}$. We will come back to this below.

So far we have focused on higher genus $g \geq 1$ Riemann surfaces. Things work in an analogous way for $S^{2}$. In this case, the chiral $q$ YM blocks given by

$$
Z_{R_{1}, R_{2}}^{q \mathrm{YM},+}=\widehat{Z}_{0} q^{\frac{k_{R_{1}}+k_{R_{2}}}{2}} e^{-\frac{t\left(\left|R_{1}\right|+\left|R_{2}\right|\right)}{(p-2)}} \sum_{R} q^{\frac{(p-2) k_{R}}{2}} e^{-t|R|} W_{R_{1} R}(q) W_{R R_{2}^{T}}(q)
$$

are computing partition functions of topological strings with two infinite stacks of D-branes in the fibers over 2 points on the $S^{2}$,

$$
Z_{R_{1} R_{2}}^{q \mathrm{YM},+}(t)=Z_{R_{1}, R_{2}}^{\mathrm{top}}(t)
$$

where the right hand side is computed using technology of section 2, up to the (ambiguous) pieces $Z^{\text {top }} \rightarrow \widehat{Z}_{0} Z^{\text {top }}$ which for us are defined in (5.16). We can write the above in an alternative way which makes contact with the topological vertex.

$Z_{R_{1} R_{2}}^{q \mathrm{YM},+}(t)=\widehat{Z}_{0} q^{\frac{k_{R_{1}}}{2}} e^{-\frac{t\left(\left|R_{1}\right|+\left|R_{2}\right|\right)}{p-2}} \sum_{R} e^{-t|R|} q^{\frac{(p-1) k_{R}}{2}} C_{0 R_{1} R^{T}}(q) C_{0 R R_{2}}(q)$,

where $C_{R_{1} R_{2} R_{3}}$ is the topological vertex defined in [5]. This is the partition function of the topological A-model on $\mathcal{O}(-p) \oplus \mathcal{O}(p-2) \rightarrow \mathbf{P}^{1}$ with noncompact Lagrangian D-branes inserted at the two of the four external lines in the web-diagram. Note that for $p=1$ the above expression for the topological string amplitude is not completely satisfactory since

$$
t=\frac{1}{2}(p-2) N g_{s}-i \theta
$$


so $R e(t) \leq 0$. The meaning of this in the black hole context is that, even though we started with $N$ D-branes, their effective charge is negative, and they really correspond to anti D-branes. In terms of the topological string, we should rewrite the amplitude in terms of the flopped geometry with the Kähler parameter given by

$$
\hat{t}=i \pi-t
$$

with $R e(\hat{t})>0$. Indeed, as we show in appendix C, we can rewrite the chiral block $Z_{R_{1}, R_{2}}^{q \mathrm{YM},+}(t)$ as follows:

$$
Z_{R_{1}, R_{2}}^{q \mathrm{YM},+}(t)=\widehat{Z}_{0}(t)(-)^{-\frac{1}{12}} e^{-\frac{\hat{t}}{12}} \sum_{R}(-)^{|R|} e^{-\hat{t}|R|} C_{R_{1} R_{2} R}(q) C_{00 R^{T}}(q) .
$$

Analogously to $g>1$ case, we can write partition sum as

$Z^{q \mathrm{YM}}\left(S^{2}\right)=\sum_{l \in \mathbf{Z}} \int d V_{1} d V_{2} Z^{q \mathrm{YM},+}\left(t+p g_{s} l ; V_{1}, V_{2}\right) Z^{q \mathrm{YM},-}\left(\bar{t}-p g_{s} l ; V_{1}^{-1}, V_{2}^{-1}\right)$

where

$$
Z^{q \mathrm{YM},+}\left(t ; V_{1}, V_{2}\right)=\sum_{R_{1}, R_{2}} Z_{R_{1}, R_{2}}^{q \mathrm{YM},+}(t) \operatorname{Tr}_{R_{1}} V_{1} \operatorname{Tr}_{R_{2}} V_{2}
$$

\subsection{Topological String Interpretation}

Above we have found that there is an apparent discrepancy between the prediction of $\mathbb{1}$ that $Z_{B H}=\left|Z^{\text {top }}\right|^{2}$ and the explicit computation of the black hole ensemble which leads to

$$
Z_{B H}=\sum_{\alpha}\left|Z_{\alpha}^{\text {top }}\right|^{2}
$$

Moreover there is an extra sum over integers: This extra sum has been explained in [2] as being related to summing over RR fluxes in the geometry (or alternatively it is required for making the chemical potential has suitable periodicity in the imaginary direction). However the extra sum over chiral blocks labelled by $\alpha$ which is given by topological string amplitudes with D-branes may appear to be in contradiction with [1]. It turns out that there is no contradiction [18], and this is related to the fact that the Calabi-Yau is non-compact and has more moduli coming from the non-compact directions. Taking these into account is equivalent to writing the black hole partition function in terms of the D-brane blocks as given above. See 18 for more detail. 


\section{The 4d Black Holes and the $c=1$ String.}

As is well known, the mirror symmetry implies that the B-model on the conifold

$$
z w-x y=\hat{t}
$$

is the same as a $\hat{t} \rightarrow 0$ limit of the A-model topological string on $O(-1) \oplus$ $O(-1) \rightarrow \mathbf{P}^{1}$, where $\hat{t}$ is the Kähler parameter of the $\mathbf{P}^{1}$. On the other hand, it is also well known that B-model topological string on the conifold is the same as the bosonic $c=1$ string at the self dual radius, where $\hat{t}$ is identified with the cosmological constant [19]. Given the results of the previous sections, we should also be able to give a non-perturbative formulation for the B-model topological string on the conifold, and hence also of the $c=1$ string theory. In other words, it follows by the mirror symmetry that the small $\hat{t}$ limit of $Z^{q \mathrm{YM}}\left(S^{2}\right)$ gives the non-perturbative formulation of the $c=1$ string.

Recall that perturbative $c=1$ amplitudes depend effectively only on the ratio $\mu$ of the cosmological constant and $g_{s}$,

$$
\mu=\frac{\hat{t}}{g_{s}}=\frac{N}{2}+i \alpha
$$

where $\alpha=(\pi-\theta) / g_{s}$. One would expect that this extends to non-perturbative contributions as well - the only finite mass D-brane in the B-model on the conifold is a D3-brane wrapping the $S^{3}$ of size $\hat{t}$, whose action is then $\mu=\frac{\hat{t}}{g_{s}}$. Given this, taking a small $\hat{t}$ limit at fixed $\mu$, is equivalent to taking to a small $g_{s}$ limit keeping $\mu$ fixed.

Consider the partition function of the $q \mathrm{YM}$ for $g=0, p=1$ obtained in section 4 ,

$Z^{q \mathrm{YM}}\left(S^{2}\right)=\left(\frac{2 \pi}{g_{s}}\right)^{N / 2} q^{\frac{N}{12}} S_{00}(q, N) \sum_{n \in \mathbf{Z}^{N}} \exp \left(-\frac{2 \pi^{2} n^{2}}{g_{s}}+\frac{2 \pi}{g_{s}} \theta \sum_{i=1}^{N} n_{i}\right)$.

The small $g_{s}$ limit of the $S_{00}\left(g_{s}, N\right)$ factor is well known to be (see [20]) given by $\frac{\left(2 \pi g_{s}\right)^{\frac{N^{2}}{2}}}{\operatorname{vol}(U(N))}\left(\frac{2 \pi}{g_{s}}\right)^{N / 2}$. Then, in the sum over instantons only the $\vec{n}=0$ sector survives in the limit, and we have a prediction for the non-perturbative partition function $Z_{c=1}$ of the $c=1$ string as

$$
Z_{c=1}=\frac{\left(2 \pi g_{s}\right)^{\frac{N^{2}}{2}}}{\operatorname{vol}(U(N))}\left(\frac{2 \pi}{g_{s}}\right)^{N}
$$


The answer suggests what is the underlying theory which provides the nonperturbative completion of $c=1$ string. It is simply the $2 \mathrm{D}$ topological YM theory. This can be seen by taking the $g_{s} \rightarrow 0$ limit of the qYM path integral. This theory should be the effective theory of $N$ D3 branes on the non-compact 3 -cycle which is dual to the $S^{3}$ of the conifold (in the same sense as $2 \mathrm{~d}$ qYM is effectively describing the $N=4$ topological YM on the D4 branes.).

In studying this theory at large $N$, we can use the results of the large $N$ limit of $q \mathrm{YM}$ theory that we already studied which led to

$$
Z^{q \mathrm{YM}}\left(S^{2}\right)=\sum_{l \in \mathbf{Z}} \sum_{R_{1}, R_{2}} Z_{R_{1}, R_{2}}^{q \mathrm{YM},+}\left(t+g_{s} l\right) Z_{R_{1}, R_{2}}^{q \mathrm{YM},-}\left(\bar{t}-g_{s} l\right)
$$

and take the $g_{s} \rightarrow 0$ limit of that, which is the same as giving a chiral decomposition of (6.3) at large $N$.

Using the results in the appendix $\mathrm{C}$, it is easy to show that

$$
A_{R_{1} R_{2}}=\lim _{g_{s} \rightarrow 0} Z_{R_{1} R_{2}}
$$

is given by

$$
A_{R_{1}, R_{2}}(\mu)=a\left(g_{s}, \mu\right) \frac{\operatorname{dim} R_{1}(\mu) \operatorname{dim} R_{2}^{T}(\mu)}{\operatorname{vol}(U(\mu))} .
$$

Here $\operatorname{dim} R(\mu)$ is given by

$$
\operatorname{dim} R(\mu)=d(R) \prod_{\square \in R}(\mu-i(\square)+j(\square))
$$

where $i, j(\square)$ label the location, i.e. the row and the column, of the box in the Young tableaux of $R$. The coefficient $d(R)$ is related to the dimension of the corresponding symmetric group representation

$$
d(R)=\prod_{\square \in R} \frac{1}{h(\square)}
$$

where $h(\square)$ is the hook length of the corresponding box in $R$. Finally, coefficient $a\left(g_{s}, \mu\right)$ is given by

$$
a\left(g_{s}, \mu\right)=\left(2 \pi g_{s}\right)^{\frac{\mu^{2}}{2}}\left(\frac{2 \pi}{g_{s}}\right)^{\mu} e^{\frac{i 2 \pi \mu^{2}}{3}} e^{i \delta}
$$


where the phase $e^{i \delta}$ is independent of $\mu$ and can be attributed to the ambiguity of the definition of the chiral block. By $\operatorname{vol}(U(\mu))$ we denote a function obtained by expanding the volume of the unitary group $U(M)$, in powers of $1 / M$, and in the result, setting $M=\mu$.

Thus, we find that, at large $N$ the non-perturbative $c=1$ partition function factorizes as

$$
Z_{c=1} \approx \sum_{l \in \mathbf{Z}} \sum_{R_{1}, R_{2}} A_{R_{1}, R_{2}}(\mu-l) A_{R_{1}^{T}, R_{2}^{T}}(\bar{\mu}+l)(-)^{\left(\left|R_{1}\right|+\left|R_{2}\right|\right)}
$$

The equality holds only in the asymptotic expansion, and we have denoted this by " $\approx "$.

It is natural to ask what the interpretation of chiral blocks $A_{R_{1} R_{2}}$ is. First of all, note that the vacuum chiral block

$$
A_{00}(\mu)=\frac{a\left(g_{s}, \mu\right)}{\operatorname{vol}(U(\mu))}
$$

in the $1 / \mu$ expansion is precisely the vacuum amplitude of the $c=1$ string. The higher chiral blocks in (6.5) are related to the scattering amplitudes of perturbative $c=1$ string at self-dual radius [21].

Recall that $c=1$ string is free when written in terms of fermions. Correspondingly, the S-matrix elements are diagonal in terms of fermions,

$$
S_{R}(\mu)=\langle R|S| R\rangle
$$

and can be brought into the form (see for example [22] 11

$$
S_{R}=\prod_{i=1}^{\# \operatorname{diag}(R)} \Gamma\left(i \mu+f_{i}+1\right) \Gamma\left(-i \mu+h_{i}+1\right) \cos \left[\frac{\pi}{2}\left(f_{i}+i \mu\right)\right] \cos \left[\frac{\pi}{2}\left(h_{i}-i \mu\right)\right]
$$

where \# $\operatorname{diag}(R)$ stands for the number of boxes on the diagonal of $R$ and the free fermion state $|R\rangle$ is expressed as:

$$
|R\rangle=\prod_{j=1}^{\# \operatorname{diag}(R)} \psi_{-\left(h_{i}+\frac{1}{2}\right)} \psi_{-\left(f_{i}+\frac{1}{2}\right)}^{*}|0\rangle
$$

11 From now on we will not be careful about constant factors like $\pi, 2$ etc 
where the fermion and hole momenta are defined as $f_{i}=R_{i}-i$ and $h_{i}=R_{i}^{T}-i$. The similarity with our blocks becomes apparent if we use

$$
\Gamma(x) \Gamma(1-x)=\frac{\pi}{\sin (\pi x)}
$$

to rewrite $S_{R}$ as

$$
\begin{aligned}
& S_{R}=\prod_{i=1}^{\# \operatorname{diag}(R)} \frac{\Gamma\left(i \mu+f_{i}+1\right)}{\Gamma\left(i \mu-h_{i}\right)} \frac{\cos \left[\frac{\pi}{2}\left(f_{i}+i \mu\right)\right] \cos \left[\frac{\pi}{2}\left(h_{i}-i \mu\right)\right]}{\sin \left[\pi\left(i \mu-h_{i}\right)\right]} \\
& =\exp \left[-\frac{i \pi}{2} \sum_{i=1}^{\# \operatorname{diag}(R)}\left(h_{i}+f_{i}\right)\right] \prod_{\square \in R}(i \mu-i(\square)+j(\square))+O\left(e^{-\mu}\right)
\end{aligned}
$$

Note that for $c=1$ string the cosmological constant $\mu$ is real, while in the topological string context $\mu$ is complex parameter. In what follows we'll take advantage of the analytic continuation provided by the topological string, and make no particular distinction between $\mu$ and $i \mu$. With this in mind, in the expression we recognize elements of $(6.5)$,

$$
S_{R}(\mu)=\exp \left[-\frac{i \pi}{2} \sum_{i=1}^{\# \operatorname{diag}(R)}\left(h_{i}+f_{i}\right)\right] \frac{\operatorname{dim} R(\mu)}{d(R)}
$$

We will express $Z_{c=1}$ as an overlap of D-brane wave functions below. This will also allow us to interpret the result as a tachyon scattering amplitude.

\section{1. $Z_{c=1}$ as an Overlap of D-Brane Wave Functions.}

It is natural to expect, given the discussion of the preceding sections, that the higher chiral blocks are computed by a particular D-brane amplitude on the B-model conifold geometry. In the $c=1$ string theory language, this would correspond to a particular tachyon scattering amplitude [22].

Moreover, we expect to be able to formulate $Z_{c=1}$, in the large $N$ expansion as an overlap of D-brane wave functions

$$
Z_{c=1}=\int \mathcal{D} x_{1} \mathcal{D} x_{2}\left|Z_{\mathrm{D}-\text { brane }}\left(x_{1}, x_{2}\right)\right|^{2}
$$


for some appropriate D-brane configuration and appropriate measure $\mathcal{D} x$ on the space of hermitian matrices of infinite rank. Using the relation of the Bmodel on the conifold with $c=1$ string, $Z_{D-\text { brane }}$ can also be thought of as a particular coherent state scattering amplitude of the $c=1$ string

$$
Z_{\mathrm{D}-\text { brane }}=Z_{\text {scatt. }} .
$$

We will show that, $Z_{\mathrm{D}-\text { brane }}\left(x_{1}, x_{2}\right)$ is a wave function in holomorphic polarization given by

$$
Z_{\text {D-brane }}\left(x_{1}, x_{2}\right)=\sum_{R_{1}, R_{2}} A_{R_{1}, R_{2}^{T}} \operatorname{Tr}_{R_{1}} x_{1} \operatorname{Tr}_{R_{2}} x_{2}
$$

Moreover there is a natural measure

$$
\mathcal{D} x=d x d \bar{x} \exp [\operatorname{tr}(x \bar{x})]
$$

over commuting Hermitian matrices $x, \bar{x}$ with the property that

$$
\int \mathcal{D} x \operatorname{Tr}_{R_{1}} x \operatorname{Tr}_{R_{2}} x=\delta_{R_{1}, R_{2}}
$$

so that (6.8) holds.

Consider $c=1$ amplitude with coherent state of incoming and outgoing tachyons turned on

$$
S(t, \bar{t})=\langle t|S| \bar{t}\rangle,
$$

where

$$
|t\rangle=\exp \left(\sum_{n=1}^{\infty} \frac{t_{n}}{n} \alpha_{-n}\right)|0\rangle,
$$

and $\alpha_{-n}$ are the usual boson creation operators corresponding here to the tachyon momentum modes. We can relate this to a D-brane correlation function in the B-model on the conifold (6.1). As explained in [22], the effective B-model theory is a theory of a chiral boson (corresponding to the tachyon) on the Riemann surface 12

$$
x \bar{x}=\mu,
$$

12 View $x, \bar{x}$ here as independent complex variables. 
and the fermions of this chiral boson are the D-branes. The Riemann surface is a cylinder with two asymptotic regions corresponding to large $x$ or large $\bar{x}$. Sending outgoing and ingoing tachyon pulses is equivalent by bosonization to placing D-branes at points $x_{i}, \bar{x}_{i}$ near one of two boundaries on the Riemann surface. The $x_{i}, \bar{x}_{i}$, viewed as eigenvalues of infinite dimensional matrices $x$ and $\bar{x}$, are related to coherent states of tachyons by

$$
t_{n}=\operatorname{tr} x^{n}, \quad \bar{t}_{n}=\operatorname{tr} \bar{x}^{n} .
$$

This provides an identification between $|t\rangle$ and D-brane configurations $|x\rangle$,

$$
|t\rangle=|x\rangle \text {. }
$$

The scattering amplitudes (6.10) are related by bosonisation to the formula (6.6) we had above. Namely, bosonisation relates

$$
|R\rangle=\sum_{\vec{k}} \frac{\chi_{R}(C(\vec{k}))}{z_{\vec{k}}}|\vec{k}\rangle,
$$

where

$$
|\vec{k}\rangle=\prod_{n}\left(\alpha_{-n}\right)^{k_{n}}|0\rangle
$$

Note that in terms of $\langle x \mid R\rangle=\operatorname{Tr}_{R} x$ and $\langle x \mid \vec{k}\rangle=\operatorname{tr}_{\vec{k}} x$ this is just the relation (5.23) where $z_{\vec{k}}$ is also defined.

From this we can write $S(t, \bar{t})$ as

$$
S(t, \bar{t})=\sum_{R} S_{R} \operatorname{Tr}_{R} x \operatorname{Tr}_{R} \bar{x}
$$

where $S_{R}$ is the scattering amplitude in (6.6). The left hand side is better thought of in terms of tachyon scattering, the right in terms of D-brane (or fermion) amplitudes. More generally, any tachyon scattering amplitude can be related to a D-brane correlation function, by bosonisation.

We will now see that

$$
\sum_{R_{1}, R_{2}} A_{R_{1}, R_{2}^{T}} \operatorname{Tr}_{R_{1}} x_{1} \operatorname{Tr}_{R_{2}} x_{2}
$$


does correspond to a scattering amplitude of the $c=1$ string, and thus to a D-brane amplitude. First of all, using properties of tensor product coefficients $N_{R_{1} R_{2}}^{R}, 13$ and the definition of $S_{Q}(\mu)$ in (6.7) it is easy to see that, up to a $\mu$-independent phase (and a trivial prefactor), the above is equal

$$
\sum_{R_{1}, R_{2}} \sum_{Q} N_{R_{1} R_{2}}^{Q} d(Q) S_{Q} \operatorname{Tr}_{R_{1}} x_{1} \operatorname{Tr}_{R_{2}} x_{2}
$$

This can be directly interpreted as a tachyon scattering amplitude as follows. First it is easy to prove 14 that

$$
\sum_{R_{1}, R_{2}, R} N_{R_{1} R_{2}}^{R} \operatorname{Tr}_{R_{1}} x_{1} \operatorname{Tr}_{R_{2}} x_{2}|R\rangle=\left|t_{1}+t_{2}\right\rangle
$$

where $\left|t_{1,2}\right\rangle$ are coherent states corresponding to $\left|x_{1}\right\rangle$ and $\left|x_{2}\right\rangle$, and we defined

$$
\left|t_{1}+t_{2}\right\rangle=\exp \left(\sum_{n} \frac{1}{n}\left(t_{1, n}+t_{2, n}\right) \alpha_{-n}\right)|0\rangle
$$

Finally, using

$$
\frac{1}{n !}\left(\alpha_{-1}\right)^{n}|0\rangle=\sum_{R} \frac{1}{n !} \chi_{R}\left(1^{n}\right)|R\rangle=\sum_{R} d(R)|R\rangle
$$

(the sum is over representations $R$ of $n$ boxes), we see that

$$
\sum_{R_{1}, R_{2}} A_{R_{1}, R_{2}^{T}} \operatorname{Tr}_{R_{1}} x_{1} \operatorname{Tr}_{R_{2}} x_{2}=\left\langle 1|S| t_{1}+t_{2}\right\rangle
$$

where

$$
|1\rangle:=\exp \left(\alpha_{-1}\right)|0\rangle
$$

To summarize, we found that $\sum_{R_{1}, R_{2}} A_{R_{1}, R_{2}^{T}} \operatorname{Tr}_{R_{1}} x_{1} \operatorname{Tr}_{R_{2}} x_{2}$ corresponds to a tachyon scattering amplitude, and thus also to a D-brane correlation function. To complete the claim that this in fact gives the D-brane or scattering amplitude

13 These satisfy $\sum_{R} N_{R_{1} R_{2}}^{R} \operatorname{Tr}_{R} x=\operatorname{Tr}_{R_{1}} x \operatorname{Tr}_{R_{2}} x$ essentially by definition, and relation we need $\sum_{R_{1}, R_{2}} N_{R_{1} R_{2}}^{Q} \operatorname{dim}(Q)=\operatorname{dim}\left(R_{1}\right) \operatorname{dim}\left(R_{2}\right)$ is a special case of this when $x$ is the identity matrix, $x=i d$.

14 For example, evaluate the above formula by contracting with arbitrary $\langle x|$ and prove that $\exp \left(\sum_{n} \frac{1}{n} \operatorname{tr} x^{n} \operatorname{tr} \bar{x}^{n}\right)=\sum_{R} \operatorname{Tr}_{R} x \operatorname{Tr}_{R} \bar{x}$. 
which computes $Z_{c=1}$, we need a natural inner product where the overlap of the above wave functions would agree with the $\frac{1}{\mu}$ expansion of $Z_{c=1}$.

In the context of tachyon scattering this is straightforward. Recall that there is a canonical measure on the space of coherent states $\psi(t)=\langle t \mid \psi\rangle$

$$
\langle\psi \mid \chi\rangle=\int \mathcal{D} t \bar{\psi}(\bar{t}) \chi(t)
$$

where

$$
\mathcal{D} t=\prod_{n=1}^{\infty} \frac{1}{n} d t_{n} d \bar{t}_{n} \exp \left(-\frac{t_{n} \bar{t}_{n}}{n}\right)
$$

This is the same inner product as the natural inner product in the $|\vec{k}\rangle$ or $|R\rangle$ basis - in other words, $\int \mathcal{D} t|t\rangle\langle\bar{t}|$ is the identity operator 15 . Putting everything together, it immediately follows

$$
Z_{c=1}=\int \mathcal{D} t_{1} \mathcal{D} t_{2}\left|Z_{\text {scatt. }}\left(t_{1}, t_{2}\right)\right|^{2}
$$

where

$$
Z_{\text {scatt. }}\left(t_{1}, t_{2}\right)=\left\langle 1|S| t_{1}+t_{2}\right\rangle
$$

The above expression is very reminiscent of the idea of [23]: The state $\langle 1$ | above is a chiral version of a black hole state. It is as if in the non-perturbative formulation of the $c=1$ theory we have been forced to consider a black hole state. Moreover the formula (6.12) is analogous to computing the decay of the black hole state to arbitrary tachyon state. It would be very interesting to develop this picture further, especially in view of the fact that we have an exact non-perturbative formulation in terms of which (6.12) is only an asymptotic large charge expansion.

We could stop here, however, this way of writing $Z_{c=1}$ is not very natural if one wants to relate it D-branes. To do this we need an inner product in the $x$-basis corresponding to D-brane positions.

$$
\langle\psi \mid \chi\rangle=\int \mathcal{D} x \bar{\psi}(\bar{x}) \chi(x)
$$

15 For example, one can easily check that $\int \mathcal{D} t\langle\vec{k} \mid t\rangle\left\langle\bar{t} \mid \vec{k}^{\prime}\right\rangle=z_{\vec{k}} \delta\left(\vec{k}, \vec{k}^{\prime}\right)$ as it should be to agree with the definition of $|\vec{k}\rangle$. 
We can use define this by asking that it is compatible with the inner product in the $R$-basis, i.e.

$$
\int \mathcal{D} x \operatorname{Tr}_{R} x \operatorname{Tr}_{Q} \bar{x}=\langle Q \mid \mathcal{R}\rangle=\delta_{Q, R}
$$

We will show in the appendix D, that the inner product exists, and can explicitly be given in terms of (6.9) as claimed above.

We have thus shown that we can alternatively write $Z_{c=1}$ in terms of Dbrane amplitude

$$
Z_{\mathrm{D}-\text { brane }}\left(x_{1}, x_{2}\right)=\sum_{R_{1}, R_{2}} A_{R_{1}, R_{2}^{T}} \operatorname{Tr}_{R_{1}} x_{1} \operatorname{Tr}_{R_{2}} x_{2}
$$

as

$$
Z_{c=1}=\int \mathcal{D} x_{1} \mathcal{D} x_{2}\left|Z_{\mathrm{D}-\text { brane }}\left(x_{1}, x_{2}\right)\right|^{2}
$$

and where

$$
Z_{\text {D-brane }}\left(x_{1}, x_{2}\right)=Z_{\text {scatt. }}\left(t_{1}, t_{2}\right)
$$

with $t_{1,2}$ and $x_{1,2}$ related by 6.11 .

\section{Acknowledgments}

We are grateful to C. Beasley, J. Bryan, M. Marino, A. Neitzke and R. Pandharipande W. Taylor and E. Witten for valuable discussions. H.O. and C.V. thank the 2004 Simons Workshop on mathematics and physics, for stimulating talks. H.O. in addition thanks the Aspen Center for Physics and the High Energy Theory Group at Harvard University for their hospitalities.

The research of M.A. was supported in part by a DOE OJI Award, and an Alfred P. Sloan Foundation fellowship. The research of H.O. was supported in part by DOE grant DE-FG03-92-ER40701. The research of N.S. and C.V. was supported in part by NSF grants PHY-0244821 and DMS-0244464. 


\section{Appendix A. The Cap Amplitude of 2d $(q)$ YM and the Fourier Trans- form}

In this appendix we compute the path integral on a disk of the ordinary $2 \mathrm{~d}$ YM theory and its $q$-deformed version, in $\Phi$ basis instead of the usual holonomy basis. In the holonomy basis, it is given by:

$$
Z_{2 \mathrm{dYM}}(C)(U)=\sum_{\mathcal{R}} \operatorname{dim}(\mathcal{R}) \operatorname{Tr}_{\mathcal{R}} U
$$

where as before $U=P e^{\oint A}$. The Fourier transform to the $\Phi$ basis is given by the following path integral over the boundary of the disk,

$$
Z_{2 \mathrm{dYM}}(C)(\Phi)=\int d U e^{\frac{1}{g_{s}} \oint_{\partial C} \operatorname{Tr} \Phi A} Z_{2 \mathrm{dYM}}(C)(U) .
$$

Since the YM path integral localizes to configurations where $\Phi$ is covariantly constant,so in particular $\Phi$ and $A$ commute, integrating over the angles gives (see [24] for details. There, effectively the same matrix integrals were considered in a related context)

$$
Z_{2 \mathrm{dYM}}(C)(\Phi)=\int \prod_{i} d u_{i} \Delta_{H}(u) e^{\frac{1}{g_{s}} \sum_{i} \vec{\phi} \cdot \vec{u}} Z_{2 \mathrm{dYM}}(C)(\vec{u}),
$$

where we defined an anti-hermitian matrix $u$ by $U=e^{u}$, and $\Delta_{H}(u)=$ $\prod_{\alpha>0} \sin (\vec{\alpha} \cdot \vec{u})$. comes from the Haar measure on $U(N)$. We can compute the integral by using

$$
\operatorname{Tr}_{\mathcal{R}} U:=\chi_{\mathcal{R}}(\vec{u})=\frac{\sum_{\omega \in w}(-1)^{\omega} e^{\omega\left(\vec{\lambda}_{\mathcal{R}}+\vec{\rho}\right) \cdot \vec{u}}}{\sum_{\omega \in w}(-1)^{\omega} e^{\omega(\vec{\rho}) \cdot \vec{u}}},
$$

where $\lambda_{\mathcal{R}}$ is the highest weight vector of the representation $\mathcal{R}$ and $\vec{\rho}$ is the Weyl vector; the Weyl denominator formula

$$
\prod_{\alpha>0} \sin (\vec{\alpha} \cdot \vec{u})=\sum_{\omega \in W}(-1)^{\omega} e^{\omega(\vec{\rho}) \cdot \vec{u}}
$$

and by writing the dimension of representation $R$ as

$$
\operatorname{dim}(\mathcal{R})=\lim _{t \rightarrow 0} \chi_{\mathcal{R}}(t \vec{\rho}) .
$$


Plugging this into the integral, and performing a sum over the weight lattice we get

$$
Z_{2 \mathrm{dYM}}(C)(\phi)=\lim _{t \rightarrow 0} \int \prod_{i} d u_{i} e^{\sum_{i} \vec{\phi} \cdot \vec{u} / g_{s}} \frac{\sum_{\omega \in W}(-1)^{\omega} \delta(\vec{u}-t \omega(\vec{\rho}))}{\prod_{\alpha>0} \sin (t \vec{\alpha} \cdot \vec{\rho})}
$$

or, by computing the integral

$$
\begin{aligned}
Z_{2 \mathrm{dYM}}(C)(\phi) & =\lim _{t \rightarrow 0} \frac{\sum_{\omega \in W}(-1)^{\omega} e^{t \vec{\phi} \cdot \omega(\vec{\rho})}}{\prod_{\alpha>0} \sin (t \vec{\alpha} \cdot \vec{\rho})} \\
& =\lim _{t \rightarrow 0} \frac{\prod_{\alpha>0} \sin (t \vec{\alpha} \cdot \vec{\phi})}{\prod_{\alpha>0} \sin (t \vec{\alpha} \cdot \vec{\rho})} .
\end{aligned}
$$

Finally, this is equal to the expression we gave in section 3

$$
Z_{2 \mathrm{dYM}}(C)(\phi)=\prod_{i<j} \frac{\phi_{i}-\phi_{j}}{i-j}
$$

up to a constant multiplicative factor $\prod_{i<j}(i-j)$ which we had dropped there.

Note that the analogue of this for the $q$-deformed amplitude,

$$
Z_{q \mathrm{YM}}=\sum_{\mathcal{R}} \operatorname{dim}_{q}(\mathcal{R}) \operatorname{Tr}_{\mathcal{R}} U
$$

is simply obtained by setting

$$
t=g_{s}
$$

in the formula for the dimension of representation (A.1), and not taking the small $t$ limit:

$$
\operatorname{dim}_{q}(\mathcal{R})=\chi_{\mathcal{R}}\left(g_{s} \vec{\rho}\right)
$$

This gives the path integral on the disk for the $q \mathrm{YM}$ theory

$$
Z_{\mathrm{qYM}}(C)(\phi)=\prod_{i<j} \frac{\left[\phi_{i} / g_{s}-\phi_{j} / g_{s}\right]_{q}}{[i-j]_{q}}
$$

as claimed in section 3 (where we needed the inverse of this Fourier transform). 
Appendix B. $\operatorname{dim}_{q}(R \bar{S})$ in terms of $K_{R S}$ or $N_{R S}$

The quantum dimension of the coupled representation has the form

$\operatorname{dim}_{q}(R \bar{S})=\operatorname{dim}_{q} R \operatorname{dim}_{q} S \prod_{i=1}^{c_{R}} \prod_{j=1}^{c_{S}} \frac{\left[S_{j}+R_{i}+N+1-j-i\right][N+1-j-i]}{\left[S_{j}+N+1-i-j\right]\left[R_{i}+N+1-j-i\right]}$,

where $c_{R}$ is the number of rows in $R$ and $R_{i}$ is the number of boxes in the i-th row. Now we let $q=e^{-g_{s}}$ and express $\operatorname{dim}_{q} R$ as

$$
\operatorname{dim}_{q} R=Q^{-\frac{|R|}{2}} W_{R}\left(q^{-1}\right) \prod_{i=1}^{c_{R}} \prod_{j=1}^{R_{i}}\left(1-q^{-i+j} Q\right), \quad Q=e^{-g_{s} N} .
$$

We write each of the products in (B.1) and (B.2)in the exponential form:

$$
\prod_{i=1}^{c_{R}} \prod_{j=1}^{c_{S}}\left(1-q^{S_{j}+R_{i}-j-i+1} Q\right)=\exp \left(-\sum_{n=1}^{\infty} \frac{g_{1}\left(q^{n}\right) Q^{n}}{n}\right),
$$

where $g_{1}(q)=\sum_{i=1}^{c_{R}} \sum_{j=1}^{c_{S}} q^{S_{j}+R_{i}-j-i+1}$.

$$
\prod_{i=1}^{c_{R}} \prod_{j=1}^{c_{S}}\left(1-q^{S_{j}-j-i+1} Q\right)=\exp \left(-\sum_{n=1}^{\infty} \frac{g_{2}\left(q^{n}\right) Q^{n}}{n}\right),
$$

where $g_{2}(q)=\sum_{i=1}^{c_{R}} \sum_{j=1}^{c_{S}} q^{S_{j}-j-i+1}$.

$$
\prod_{i=1}^{c_{R}} \prod_{j=1}^{c_{S}}\left(1-q^{R_{i}-j-i+1} Q\right)=\exp \left(-\sum_{n=1}^{\infty} \frac{g_{3}\left(q^{n}\right) Q^{n}}{n}\right)
$$

where $g_{3}(q)=\sum_{i=1}^{c_{R}} \sum_{j=1}^{c_{S}} q^{R_{i}-j-i+1}$.

$$
\prod_{i=1}^{c_{R}} \prod_{j=1}^{c_{S}}\left(1-q^{-j-i+1} Q\right)=\exp \left(-\sum_{n=1}^{\infty} \frac{g_{4}\left(q^{n}\right) Q^{n}}{n}\right),
$$

where $g_{4}(q)=\sum_{i=1}^{c_{R}} \sum_{j=1}^{c_{S}} q^{-j-i+1}$.

$$
\prod_{i=1}^{c_{R}} \prod_{j=1}^{R_{i}}\left(1-q^{-i+j} Q\right)=\exp \left(-\sum_{n=1}^{\infty} \frac{f_{R}\left(q^{n}\right) Q^{n}}{n}\right),
$$

where $f_{R}(q)=\sum_{i=1}^{c_{R}} \sum_{j=1}^{R_{i}} q^{-i+j}$. 
So that $\operatorname{dim}_{q}(R \bar{S})$ is re-casted as

$$
\operatorname{dim}_{q}(R \bar{S})=Q^{-\frac{|R|+|S|}{2}} W_{R}\left(q^{-1}\right) W_{S}\left(q^{-1}\right) \exp \left(-\sum_{n=1}^{\infty} \frac{M_{R S}\left(q^{n}\right) Q^{n}}{n}\right)
$$

where $M_{R S}(q)=g_{1}(q)+g_{4}(q)-g_{2}(q)-g_{3}(q)+f_{R}(q)+f_{S}(q)$.

It turns out that $M_{R S}(q)=f_{R S}(q)$ where

$$
f_{R S}(q)=\left(q-2+q^{-1}\right) f_{R}(q) f_{S}(q)+f_{R}(q)+f_{S}(q) .
$$

Now, we relate $\operatorname{dim}_{q}(R \bar{S})$ with the functions $K_{R S}(q)$ and $N_{R S}(q)$ defined as follows:

$$
\begin{gathered}
K_{R S}(Q, q):=\sum_{P} Q^{|P|} W_{P R}(q) W_{P S}(q)=W_{R}(q) W_{S}(q) \prod_{i=1, j=1}^{\infty}\left(1-Q x_{i} y_{j}\right)^{-1} \\
N_{R S}(Q, q):=\sum_{P}(-)^{|P|} Q^{|P|} W_{P R}(q) W_{P^{T} S}(q)=W_{R}(q) W_{S}(q) \prod_{i=1, j=1}^{\infty}\left(1-Q x_{i} y_{j}\right),
\end{gathered}
$$

where $x_{i}=q^{R_{i}-i+1 / 2}, \quad y_{j}=q^{S_{j}-j+1 / 2}$. We used the definition of $W_{R_{1} R_{2}}$ in terms of Schur functions $s_{R}$ :

$$
W_{P R}(q)=s_{R}\left(q^{-i+1 / 2}\right) s_{P}\left(q^{R_{i}-i+1 / 2}\right)
$$

and the properties of Schur functions

$$
\sum_{R} s_{R}(x) s_{R}(y)=\prod_{i, j}\left(1-x_{i} y_{j}\right)^{-1}, \quad \sum_{R} s_{R}(x) s_{R^{T}}(y)=\prod_{i, j}\left(1+x_{i} y_{j}\right) .
$$

As follows from [25] the functions $K_{R S}$ and $N_{R S}$ are expressed in terms of $f_{R S}(q)$ as

$$
\begin{gathered}
K_{R S}(Q, q)=K_{. .}(Q, q) W_{R}(q) W_{S}(q) e^{\sum_{n=1}^{\infty} \frac{f_{R S}\left(q^{n}\right) Q^{n}}{n}}, \\
N_{R S}(Q, q)=N_{. .}(Q, q) W_{R}(q) W_{S}(q) e^{-\sum_{n=1}^{\infty} \frac{f_{R S}\left(q^{n}\right) Q^{n}}{n}},
\end{gathered}
$$

where we denoted the trivial representation with $R=$. and where $N_{. .}(Q, q)=$ $(K . .(Q, q))^{-1}$.

Now using $W_{R}\left(q^{-1}\right)=(-)^{|R|} W_{R^{T}}(q)=(-)^{|R|} q^{-\frac{k_{R}}{2}} W_{R}(q)$ we find the relations

$$
\operatorname{dim}_{q}(R \bar{S})=K_{. .}(Q, q) N_{R S}(Q, q)(-)^{|R|+|S|} q^{-\frac{k_{R}+k_{S}}{2}} Q^{-\frac{|R|+|S|}{2}}
$$




$$
\operatorname{dim}_{q}(R \bar{S})=\frac{K_{. .}(Q, q)}{K_{R S}(Q, q)} W_{R}^{2}(q) W_{S}^{2}(q)(-)^{|R|+|S|} q^{-\frac{k_{R}+k_{S}}{2}} Q^{-\frac{|R|+|S|}{2}} .
$$

Finally, we use that

$$
q^{\rho^{2}+\frac{N}{24}} S_{00}=M(q) \eta^{N}(q) N_{. .}(Q, q)
$$

to obtain the relations used in section 5:

$$
\begin{gathered}
q^{\rho^{2}+\frac{N}{24}} S_{00} \operatorname{dim}_{q}(R \bar{S})=M(q) \eta^{N}(q) N_{R S}(Q, q)(-)^{|R|+|S|} q^{-\frac{k_{R}+k_{S}}{2}} Q^{-\frac{|R|+|S|}{2}} \\
q^{\rho^{2}+\frac{N}{24}} S_{00} \operatorname{dim}_{q}(R \bar{S})=\frac{M(q) \eta^{N}(q)}{K_{R S}(Q, q)} W_{R}^{2}(q) W_{S}^{2}(q)(-)^{|R|+|S|} q^{-\frac{k_{R}+k_{S}}{2}} Q^{-\frac{|R|+|S|}{2}} .
\end{gathered}
$$

\section{Appendix C. Expressing $p=1, g=0$ Chiral Blocks in terms of S- Matrix.}

Below we compute the genus $\mathrm{g}=0, \mathrm{p}=1$ chiral block in terms of S-matrix. Let us define $t_{K}$ as $t=i \pi+t_{K}$ and write the chiral block as

$$
Z_{R_{1}, R_{2}}^{q \mathrm{YM},+}(t)=\widehat{Z}_{0} e^{t\left(\left|R_{1}\right|+\left|R_{2}\right|\right)} q^{\frac{k_{R_{1}}}{2}} Z_{R_{1} R_{2}}^{\prime}\left(t_{K}\right),
$$

where

$$
Z_{R_{1} R_{2}}^{\prime}\left(t_{K}\right):=\sum_{R}(-)^{|R|} e^{-t_{K}|R|} C_{0 R_{1} R^{T}}(q) C_{0 R R_{2}}(q)
$$

First, we use the definition of the vertex in terms of Schur functions

$$
C_{R_{1} R_{2} R_{3}}=q^{\frac{k_{R_{1}}}{2}} s_{R_{3}}\left(q^{\rho}\right) \sum_{\eta} s_{R_{1}^{T} / \eta}\left(q^{R_{3}+\rho}\right) s_{R_{2} / \eta}\left(q^{R_{3}^{T}+\rho}\right)
$$

to recast the $\operatorname{sum} Z_{R_{1} R_{2}}^{\prime}\left(t_{K}\right)$ as

$$
Z_{R_{1} R_{2}}^{\prime}\left(t_{K}\right)=(-)^{\left|R_{2}\right|} s_{R_{1}}\left(q^{\rho}\right) \sum_{R} s_{R}\left(\lambda q^{\rho+R_{1}}\right) \sum_{\eta} s_{R / \eta}\left(q^{-\rho}\right) s_{R_{2}^{T} / \eta}\left(q^{-\rho}\right)
$$

where $\rho^{i}=-i+\frac{1}{2}, \quad i=1, \ldots, \infty$ and $\lambda=e^{-t_{K}}$.

Next, we use the identities

$$
\sum_{R} s_{R}\left(\lambda q^{\rho+R_{1}}\right) s_{R / \eta}\left(q^{-\rho}\right)=s_{\eta}\left(\lambda q^{\rho+R_{1}}\right) \prod_{i, j}\left(1-\lambda q^{R_{1}^{i}-i+j}\right)^{-1}
$$


and

$$
\sum_{\eta} s_{R_{2}^{T} / \eta}\left(q^{-\rho}\right) s_{\eta}\left(\lambda q^{\rho+R_{1}}\right)=\lambda^{\left|R_{2}\right|} s_{R_{2}^{T}}\left(\lambda^{-1} q^{-\rho}, q^{\rho+R_{1}}\right)
$$

and bring $Z_{R_{1} R_{2}}^{\prime}\left(t_{K}\right)$ into the form

$$
Z_{R_{1} R_{2}}^{\prime}\left(t_{K}\right)=(-)^{\left|R_{2}\right|} s_{R_{1}}\left(q^{\rho}\right) \prod_{i, j}\left(1-\lambda q^{R_{1}^{i}-i+j}\right)^{-1} \lambda^{\left|R_{2}\right|} s_{R_{2}^{T}}\left(\lambda^{-1} q^{-\rho}, q^{\rho+R_{1}}\right) .
$$

This can be further simplified by using

$$
\prod_{i, j}\left(1-\lambda q^{R_{1}^{i}-i+j}\right)^{-1}=\prod_{i, j}\left(1-\lambda q^{-i+j}\right)^{-1} \prod_{i=1}^{c_{R_{1}}} \prod_{j=1}^{R_{1}^{i}}\left(1-\lambda q^{-i+j}\right) .
$$

Now we recall the formula for quantum dimension

$$
\operatorname{dim}_{q} R_{1}(q, \lambda)=\lambda^{-\frac{\left|R_{1}\right|}{2}} s_{R_{1}}\left(q^{-\rho}\right) \prod_{i=1}^{c_{R_{1}}} \prod_{j=1}^{R_{1}^{i}}\left(1-\lambda q^{-i+j}\right)
$$

and compare $Z_{R_{1} R_{2}}^{\prime}\left(t_{K}\right)$ with $W_{R_{1} R_{2}^{T}}(q, \lambda):=\frac{S_{R_{1} R_{2}^{T}}}{S_{00}}$

$$
W_{R_{1} R_{2}^{T}}(q, \lambda)=\operatorname{dim}_{q} R_{1}(q, \lambda) \lambda^{\frac{\left|R_{2}\right|}{2}} s_{R_{2}^{T}}\left(\lambda^{-1} q^{-\rho}, q^{\rho+R_{1}}\right) .
$$

We use $s_{R_{1}}\left(q^{\rho}\right)=(-)^{\left|R_{1}\right|} q^{-\frac{k_{R_{1}}}{2}} s_{R_{1}}\left(q^{-\rho}\right)$ to find

$$
Z_{R_{1} R_{2}}^{\prime}\left(t_{K}\right)=(-)^{\left|R_{1}\right|+\left|R_{2}\right|} e^{-\frac{t_{K}}{2}\left(\left|R_{1}\right|+\left|R_{2}\right|\right)} q^{-\frac{k_{R}}{2}} N_{. .}(\lambda, q) W_{R_{1} R_{2}^{T}}(q, \lambda),
$$

where $\lambda=e^{-t_{K}}, \quad N_{. .}(\lambda, q)=\prod_{i, j}\left(1-\lambda q^{-i+j}\right)^{-1}$. The chiral block is then expressed in terms of $W_{R_{1} R_{2}^{T}}$ as follows

$$
Z_{R_{1}, R_{2}}^{q \mathrm{YM},+}(t)=\widehat{Z}_{0}(t) N_{. .}(\lambda, q) e^{\frac{t_{K}}{2}\left(\left|R_{1}\right|+\left|R_{2}\right|\right)} W_{R_{1} R_{2}^{T}}(q, \lambda)
$$

where $t=i \pi+t_{K}$.

If we now let $\hat{t}=-t_{K}$ and express $W_{R_{1} R_{2}^{T}}(q, \lambda)$ as in 26

$$
W_{R_{1} R_{2}^{T}}(q, \lambda)=\frac{e^{\frac{\hat{t}}{2}\left(\left|R_{1}\right|+\left|R_{2}\right|\right)}}{N\left(e^{-\hat{t}}, q\right)} \sum_{R}(-)^{|R|} e^{-\hat{t}|R|} C_{R_{1} R_{2} R}(q) C_{00 R^{T}}(q),
$$

we find the "flopped" expression for the chiral block:

$$
Z_{R_{1}, R_{2}}^{q \mathrm{YM},+}(t)=\widehat{Z}_{0}(t) \frac{N\left(e^{\hat{t}}, q\right)}{N\left(e^{-\hat{t}}, q\right)} \sum_{R}(-)^{|R|} e^{-\hat{t}|R|} C_{R_{1} R_{2} R}(q) C_{00 R^{T}}(q) .
$$


The final step is to use the relation [26]

$$
N(Q, q)=N\left(Q^{-1}, q\right)(-Q)^{-\frac{1}{12}}
$$

to bring the flopped chiral block into the form:

$$
Z_{R_{1}, R_{2}}^{q \mathrm{YM},+}(t)=\widehat{Z}_{0}(t)\left(-e^{\hat{t}}\right)^{-\frac{1}{12}} \sum_{R}(-)^{|R|} e^{-\hat{t}|R|} C_{R_{1} R_{2} R}(q) C_{00 R^{T}}(q) .
$$

\section{Appendix D. The Inner Product of D-Brane Wave Functions in $c=1$ String}

Here we show that the matrix integral

$$
\int \mathcal{D} x \operatorname{Tr}_{R} x \operatorname{Tr}_{Q} \bar{x}=\int d x d \bar{x} \exp [\operatorname{tr}(x \bar{x})] \operatorname{Tr}_{R} x \operatorname{Tr}_{Q} \bar{x} .
$$

in the definition of the $c=1$ string overlap equals

$$
\int \mathcal{D} x \operatorname{Tr}_{R} x \operatorname{Tr}_{Q} \bar{x}=\delta_{Q, R} \text {. }
$$

This is a so called "normal" matrix integral, meaning that $x$ and $\bar{x}$ are commuting matrices.

We will begin with an analogous finite $M$ integral and than take $M$ to infinity. Integrating over the angles in the above formula is standard, where one gets

$$
\frac{1}{M !} \int \prod_{i} d x_{i} d y_{i} \Delta(x) \Delta(\bar{x}) \exp [\operatorname{tr}(x \bar{x})] \operatorname{Tr}_{R} x \operatorname{Tr}_{Q} \bar{x}
$$

It is useful here to change variables to $x$ and $z$ where

$$
\bar{x}=z / x,
$$

which gives

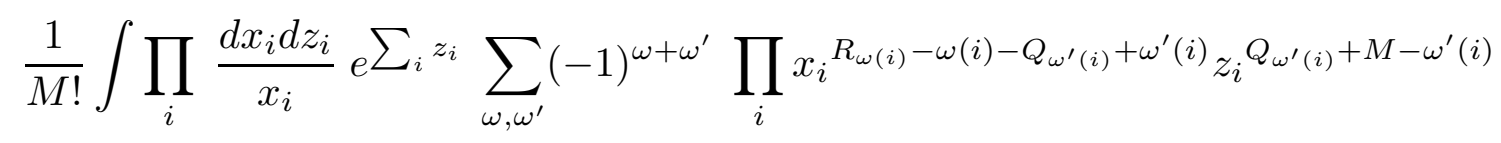

where we have in addition used the trace formula

$$
\operatorname{Tr}_{R} A=\frac{\operatorname{det}_{i, j}\left(A_{i}^{R_{j}+M-j}\right)}{\Delta(A)},
$$


which holds for any matrix $A$, and written

$$
\operatorname{det}\left(A_{i j}\right)=\sum_{\omega}(-1)^{\omega} A_{1 \omega(1)} \ldots A_{M \omega(M)}
$$

Integrating over $x_{i}$ gives zero, unless $R=Q$ and $\omega=\omega^{\prime}$ and we are left with computing

$$
\prod_{i} \int d z_{i} e^{z_{i}} z_{i}{ }^{Q_{i}+M-i}=\prod_{i=1}^{M}\left(Q_{i}+M-i\right) !
$$

All in all this gives, for $\operatorname{rank} M$ matrix

$$
\frac{1}{\operatorname{vol}(U(M))} \int d x d \bar{x} \exp [\operatorname{tr}(x \bar{x})] \operatorname{Tr}_{R} x \operatorname{Tr}_{Q} \bar{x} \propto \frac{(2 \pi)^{\frac{M^{2}}{2}+\frac{M}{2}}}{\operatorname{vol}(U(M))} \frac{\operatorname{dim}(Q)}{d(Q)} \delta_{R, Q} .
$$

Taking $M \rightarrow \infty$ limit of this corresponding to matrices of infinite rank gives

$$
\lim _{M \rightarrow \infty} \int d x d \bar{x} \exp [\operatorname{tr}(x \bar{x})] \operatorname{Tr}_{R} x \operatorname{Tr}_{Q} \bar{x}=\delta_{R, Q}
$$

where we used that, for large $M$ the dimension of $S U(M)$ representation $\operatorname{dim}(R)$ becomes the dimension of the corresponding symmetric group representation $d(R)$ (up to an infinite factor $M^{|R|}$ which we absorb in $x, \bar{x}$ and factors such as $\pi^{M}$ which go into renormalizing the measure). 


\section{References}

[1] H. Ooguri, A. Strominger, and C. Vafa, "Black hole attractors and the topological string," Phys. Rev. D70 (2004) 106007, hep-th/0405146.

[2] C. Vafa, "Two-dimensional Yang-Mills, black holes and topological strings," hep-th/0406058.

[3] D. Gross and W. Taylor, "Two-dimensional QCD is a string theory," Nucl. Phys. B400 (1993) 181-210, hep-th/9301068; "Twists and Wilson loops in the string theory of two-dimensional QCD," Nucl. Phys. B403 (1993) 395-452, hep-th/9303046.

[4] J. Bryan and R. Pandharipande, "Local Gromov-Witten theory of curves," math.AG/0411037.

[5] M. Aganagic, A. Klemm, M. Marino, and C. Vafa, "The topological vertex," hep-th/0305132.

[6] E. Witten, "Two-dimensional gauge theories revisited," J. Geom. Phys. 9 (1992) 303, hep-th/9204083.

[7] A. Iqbal, N. Nekrasov, A. Okounkov, and C. Vafa, "Quantum foam and topological strings," hep-th/0312022.

[8] N. Nekrasov, "Seiberg-Witten prepotential from instanton counting," Adv. Theor. Math. Phys. 7 (2004) 831, hep-th/0206161.

[9] E. Buffenoir and P. Roche, "Two-dimensional lattice gauge theory based on a quantum group," Commun. Math. Phys. 170, 669 (1995) arXiv:hepth/9405126.

[10] C.Klimcik, "The formulae of Kontsevich and Verlinde from the perspective of the Drinfeld double," Commun. Math. Phys. 217 (2001) 203-228, hepth/9911239.

[11] M. Blau and G. Thompson, "Derivation of the Verlinde formula from Chern-Simons theory and the $G / G$ model," Nucl. Phys. B408 (1993) 345, hep-th/9305010.

[12] C. Beasley and E. Witten, private communication.

[13] E. Witten, "Quantum field theory and the Jones polynomial," Com. Math. Phys. 121 (1989) 351.

[14] C. Vafa and E. Witten, "A strong coupling test of S-duality," Nucl. Phys. B 431 (1994) 3, hep-th/9408074.

[15] R. Rudd, "String partition function for QCD on the torus," hep-th/9407176.

[16] K. Yoshioka, "Betti numbers of moduli of stable sheaves on some surfaces," Proceedings of ICTP Conference S-Duality and Mirror Symmetry (Trieste, 1995), Nucl. Phys. B Proc. Suppl. 46 (1996) 263. 
[17] H. Nakajima, "Instantons on ALE spaces, quiver varieties and Kac-Moody algebras," Duke Math. J. 76 (1994) 365.

[18] M. Aganagic, A. Neitzke, C. Vafa, to appear

[19] D. Ghoshal and C. Vafa, " $c=1$ string as the topological theory of the conifold," Nucl. Phys. B 453 (1995) 121, hep-th/9506122.

[20] H. Ooguri and C. Vafa, "Worldsheet derivation of large $N$ duality," Nucl. Phys. B641 (2002) 3, hep-th/0205297.

[21] R. Dijkgraaf, G. Moore, R. Plesser, "The partition function of $2 \mathrm{~d}$ string theory," Nucl. Phys. B394 (1993) 356, hep-th/9208031.

[22] M. Aganagic, R. Dijkgraaf, A. Klemm, M. Marino and C. Vafa, "Topological strings and integrable hierarchies," hep-th/0312085.

[23] V. Kazakov, I. K. Kostov and D. Kutasov, "A matrix model for the twodimensional black hole," Nucl. Phys. B 622 (2002) 141, hep-th/0101011.

[24] M. Aganagic, A. Klemm, M. Marino and C. Vafa, "Matrix model as a mirror of Chern-Simons theory," JHEP 0402, 010 (2004) arXiv:hepth/0211098.

[25] A. Iqbal and A. Kashani-Poor, "Instanton counting and Chern-Simons theory," Adv. Theor. Math. Phys. 7 (2003) 459-499, hep-th/0212279.

[26] A. Iqbal and A. Kashani-Poor, "The vertex on a strip," hep-th/040174. 